\title{
Microglial phenotypes and toll-like receptor 2 in the substantia nigra and hippocampus of incidental Lewy body disease cases and Parkinson's disease patients
}

\author{
Karlijn J Doorn ${ }^{1,2}$, Tim Moors², Benjamin Drukarch², Wilma DJ van de Berg², Paul J Lucassen
} and Anne-Marie van Dam²*

\begin{abstract}
Next to a-synuclein deposition, microglial activation is a prominent pathological feature in the substantia nigra (SN) of Parkinson's disease (PD) patients. Little is known, however, about the different phenotypes of microglia and how they change during disease progression, in the SN or in another brain region, like the hippocampus (HC), which is implicated in dementia and depression, important non-motor symptoms in PD.

We studied phenotypes and activation of microglia in the SN and HC of established PD patients (Braak PD stage 4-6), matched controls (Braak PD stage 0) and of incidental Lewy Body disease (iLBD) cases (Braak PD stage 1-3) that are considered a prodromal state of PD. As recent experimental studies suggested that toll-like receptor 2 (TLR2) mediates a-synuclein triggered microglial activation, we also studied whether TLR2 expression is indeed related to pathology in iLBD and PD patients.

A clear a-synuclein pathology-related increase in amoeboid microglia was present in the HC and SN in PD. Also, morphologically primed/reactive microglial cells, and a profound increase in microglial TLR2 expression were apparent in iLBD, but not PD, cases, indicative of an early activational response to PD pathology. Moreover, TLR2 was differentially expressed between the $\mathrm{SN}$ and $\mathrm{HC}$, consistent with a region-specific pattern of microglial activation. In conclusion, the regional changes in microglial phenotype and TLR2 expression in primed/reactive microglia in the SN and HC of iLBD cases indicate that TLR2 may play a prominent role in the microglial-mediated responses that could be important for PD progression.
\end{abstract}

Keywords: Parkinson's disease, Substantia nigra, Hippocampus, Incidental Lewy body disease, Microglia, Toll-like receptor 2

\section{Introduction}

Parkinson's disease (PD) is a progressive neurodegenerative disorder that affects $1-2 \%$ of the elderly population [1]. Besides classical motor problems that are related to nigro-striatal dopamine deficits [2], also nonmotor symptoms are common in PD. These include autonomic dysfunction, sensory, sleep and cognitive disturbances as well as neuropsychiatric alterations, that

\footnotetext{
*Correspondence: amw.vandam@vumc.nl

${ }^{2}$ Department Anatomy and Neurosciences, VU University Medical Center, Neuroscience Campus Amsterdam, Van der Boechorststraat 7, 1081 BT, Amsterdam, The Netherlands

Full list of author information is available at the end of the article
}

strongly affect the quality of life of PD patients [3-8]. An important pathological hallmark of PD is the presence of $\alpha$-synuclein inclusion bodies, i.e. Lewy bodies (LBs) and Lewy neurites (LNs), which spread in a predictable manner throughout the brain [9]. While their spatiotemporal development parallels the appearance of non-motor and motor symptoms [10], little is known about changes in microglial activation, another prominent pathological feature of PD [11-13].

Under neurodegenerative conditions, microglial cells readily transform from a ramified morphology into amoeboid-shaped cells. They acquire specific functions, including phagocytosis, and can secrete a variety of factors, 
such as cytokines, chemokines, reactive oxygen species (ROS) and trophic factors [14,15]. While in vitro and experimental animal studies had demonstrated that $\alpha$ synuclein can trigger microglial activation, in vivo imaging and post-mortem immunohistochemical studies have now established the presence of amoeboid microglia and proinflammatory mediators in the substantia nigra (SN) in PD [11,16-20]. Interestingly, increased densities of amoeboid microglia were also observed in the olfactory bulb (OB) of PD patients and in a mouse model for PD (1-methyl4-phenyl-1,2,3,6-tetrahydropyridine) [21,22,23,24]. Thus, microglial activation occurs also outside the $\mathrm{SN}$, where it coincides with $\alpha$-synuclein deposition. In contrast to the $\mathrm{SN}$, it is not associated with neuronal cell death [23]. These observations agree with novel views on microglia as a heterogeneous cell population that may exert brain regiondependent functions [13,25,26,27]. As such, microglia are thought to contribute to local inflammatory responses, not in a uniform, but rather in a brain region-specific manner.

Based on their morphology and receptor expression, different microglial phenotypes have been identified in the brain $[28,29]$. These include cells with a small cell body and many thin ramifications, called ramified microglia, cells with a larger, less round cell body and thick ramifications, called primed and reactive microglia, and cells with hardly or no ramifications, which are classified as amoeboid microglia [29]. Besides these different morphologies, that likely reflect different functionalities [30-32], also toll-like receptors (TLR) have attracted considerable attention. TLR activation of microglia forms the primary response against a wide array of pathogens $[33,34]$, including aggregated proteins, e.g. $\alpha$-synuclein $[34,35]$. Activation of the TLR pathway increases the expression of various pro-inflammatory cytokines, like IL-6, IL-1 and TNF- $\alpha[36,37]$.While earlier evidence has been obtained in the field of Alzheimer's disease (AD) $[38,39]$ and Multiple Sclerosis (MS) [40], recent studies have implicated TLRs also in the pathogenesis of PD [41-44]. In support of this, TLR-deficient mice are less vulnerable to MPTP toxicity and show decreased microglial activation in the SN after MPTP treatment [45], while (Thy1)[A30P] $\alpha$-synuclein transgenic mice show a significant upregulation of TLR2 [46]. Moreover, the oligomeric form of $\alpha$-synuclein acts as an endogenous agonist of TLR2 on microglia where it stimulates pro-inflammatory cytokine expression [47]. As this effect is not induced by TLR3 and TLR4, the TLR2 subtype is considered highly specific for the neuroinflammatory response of microglia in PD [41].

Thus far, studies on microglia in PD have focused mainly on the SN. Based on the spatiotemporal development of $\alpha$-synuclein pathology over different brain regions during $\mathrm{PD}$ progression, also microglial activation may differ between brain regions. In the present study, we investigated differences in microglial phenotype, i.e. in ramified, primed/reactive and amoeboid subtypes, and compared the SN to the hippocampus (HC), a brain region that has been implicated in the cognitive deficits and depressive symptoms frequently present in PD $[48,49]$. We studied tissue of clinically diagnosed and neuropathologically verified PD patients (Braak PD stage 4-6) [50], age- and gender-matched control subjects (Braak PD stage 0) and incidental Lewy body disease (iLBD) cases (Braak PD 1-3), that did not have clinical PD symptoms but displayed $\alpha$-synuclein deposition at autopsy and can therefore be considered a prodromal state of PD [51]. Moreover, as $\alpha$-synuclein can stimulate microglia through TLR2, we further investigated whether TLR2 is expressed in the SN and HC of iLBD and PD cases in relation to $\alpha$-synuclein deposition.

\section{Materials \& methods}

\section{Post-mortem brain tissue}

Human post-mortem brain tissue was obtained from the Netherlands Brain Bank (NBB, Amsterdam, The Netherlands) or from the department of Pathology, VU University Medical Center (VUmc, Amsterdam, The Netherlands). In compliance with all local ethical and legal guidelines, informed consent for brain autopsy and the use of brain tissue and clinical information for scientific research was given by either the donor or the next of kin. The $\mathrm{SN}$ and $\mathrm{HC}$ were included from 14 clinically diagnosed and neuropathologically verified PD patients (Braak PD stage 4-6). Of these, $8 \mathrm{SN}$ and $9 \mathrm{HC}$ were included from patients who had suffered from dementia during PD progression (PDD). Furthermore, from iLBD cases (Braak PD stage 1-3), 9 SN and 6 HC were studied. The control group consisted of healthy subjects without neurological or psychiatric disease and without LB pathology (Braak PD stage 0), of which $13 \mathrm{SN}$ and $9 \mathrm{HC}$ were studied. Sections from all donors were included in the studies performed. Importantly, in $75 \%$ of the cases, the $\mathrm{HC}$ and $\mathrm{SN}$ tissue was obtained from the same patient, ruling out the possibility that regional differences are due to 'between patient' differences. All three groups were matched for gender and age; control subjects ranged from 62-92, iLBD cases from 56-91 and PD patients ranged from 59-96 years of age. Furthermore, all subjects were controlled and matched for post-mortem delay and cerebrospinal fluid $\mathrm{pH}$ value. Donors who died of sepsis, or stroke were excluded (Table 1).

The Braak neurofibrillary tangles (NFT) stages and $A \beta$ scores for $\mathrm{AD}$, and $\mathrm{LBs} / \mathrm{LNs}$ containing $\alpha$-synuclein score for PD were provided by the NBB and were based upon careful neuropathological evaluation of diseaserelevant brain areas by established and qualified neuropathologists [52]. The density and distribution of LBs/ LNs, NFT and A $\beta$ plaques were determined based on Bodian silver staining and immunohistochemical analysis 
Table 1 Clinical and neuropathological information of all included subjects

\begin{tabular}{|c|c|c|c|c|c|c|c|c|}
\hline \multirow[b]{2}{*}{ C\# } & \multirow[b]{2}{*}{ Sex } & \multirow[b]{2}{*}{ Age } & \multirow[b]{2}{*}{ PMD (hrs) } & \multicolumn{2}{|l|}{ Braak staging } & \multirow[b]{2}{*}{ D } & \multirow[b]{2}{*}{ Region } & \multirow[b]{2}{*}{ Cause of death } \\
\hline & & & & $A D(N F T / A \beta)$ & PD (a-syn) & & & \\
\hline 1 & $\mathrm{~F}$ & 92 & 7:00 & $1 \mathrm{~A}$ & 0 & NDC & $\mathrm{HC}+\mathrm{SN}$ & Acute death, pulmonary emboly \\
\hline 2 & M & 88 & 4:23 & $2 \mathrm{~A}$ & 0 & NDC & $\mathrm{HC}+\mathrm{SN}$ & Gastro-intestinal bleeding \\
\hline 3 & $\mathrm{~F}$ & 84 & $6: 55$ & 10 & 0 & NDC & $\mathrm{HC}+\mathrm{SN}$ & Myelodysplasia \\
\hline 4 & M & 82 & 5:10 & 10 & 0 & NDC & $\mathrm{HC}+\mathrm{SN}$ & Unknown \\
\hline 5 & M & 62 & $7: 20$ & 10 & 0 & NDC & $\mathrm{HC}+\mathrm{SN}$ & Unknown \\
\hline 6 & $\mathrm{~F}$ & 77 & $2: 55$ & 1B & 0 & NDC & SN & Pulmonary metastasis \\
\hline 7 & M & 84 & $5: 35$ & $1 \mathrm{~A}$ & 0 & NDC & SN & Heart failure \\
\hline 8 & M & 75 & 4:15 & 1B & 0 & NDC & SN & Respiratory insufficiency \\
\hline 9 & M & 71 & $6: 00$ & $2(-)$ & 0 & NDC & SN & Respiratory insufficiency \\
\hline 10 & M & 81 & 7:55 & 20 & 0 & NDC & SN & Renal insufficiency \\
\hline 11 & $\mathrm{~F}$ & 78 & $7: 30$ & $2(-)$ & 0 & NDC & SN & Heart failure \\
\hline 12 & M & 79 & $6: 00$ & 1B & 0 & NDC & SN & Metastates prostate and lung cancer \\
\hline 13 & $\mathrm{~F}$ & 83 & 8:00 & 10 & 0 & NDC & SN & Respitatory insuffisiency \\
\hline 14 & $\mathrm{~F}$ & 83 & $3: 20$ & $1 \mathrm{~B}$ & 0 & NDC & $\mathrm{HC}$ & Legal Euthanasia \\
\hline 15 & $\mathrm{~F}$ & 84 & 4:45 & 10 & 0 & NDC & $\mathrm{HC}$ & Heart failure \\
\hline 16 & $\mathrm{~F}$ & 85 & $5: 19$ & $2 B$ & 0 & NDC & $\mathrm{HC}$ & Natural death, pulmonary disease \\
\hline 17 & M & 78 & $<17: 00$ & 10 & 0 & NDC & $\mathrm{HC}$ & Natural cause, \\
\hline 18 & $\mathrm{~F}$ & 82 & $5: 10$ & 20 & 2 & iLBD & $\mathrm{HC}+\mathrm{SN}$ & Heartfailure \\
\hline 19 & M & 86 & 4:00 & $2 B$ & 1 & iLBD & $\mathrm{HC}+\mathrm{SN}$ & Respiratory insufficiency \\
\hline 20 & M & 56 & $5: 00$ & $0(-)$ & 2 & iLBD & $\mathrm{HC}+\mathrm{SN}$ & Pneunomia \\
\hline 21 & $\mathrm{~F}$ & 91 & 4:50 & $(-)$ & 1 & iLBD & $\mathrm{HC}+\mathrm{SN}$ & Exhaustion, colon carcinoma \\
\hline 22 & M & 84 & $7: 20$ & $1 \mathrm{~B}$ & 3 & iLBD & $\mathrm{HC}+\mathrm{SN}$ & Prostate cancer \\
\hline 23 & $\mathrm{~F}$ & 82 & 7:00 & 10 & 3 & iLBD & SN & Pneunomia \\
\hline 24 & $\mathrm{~F}$ & 85 & $4: 40$ & $2 \mathrm{~A}$ & 1 & iLBD & SN & Dehydration \\
\hline 25 & $\mathrm{~F}$ & 93 & $<10: 00$ & $0(-)$ & 1 & iLBD & SN & Unknown \\
\hline 26 & $\mathrm{~F}$ & 78 & $<10: 00$ & $0(-)$ & 1 & iLBD & SN & Unknown \\
\hline 27 & M & 87 & $10: 20$ & $1 \mathrm{~A}$ & 1 & iLBD & $\mathrm{HC}$ & Pneunomia, heart failure \\
\hline 28 & M & 83 & 4:50 & $1 \mathrm{~A}$ & 4 & PDD & $\mathrm{HC}+\mathrm{SN}$ & Heartfailure \\
\hline 29 & $\mathrm{~F}$ & 59 & $9: 35$ & $1 \mathrm{~A}$ & 4 & PD & $\mathrm{HC}+\mathrm{SN}$ & Shock due to blood loss in digestive tract \\
\hline 30 & $\mathrm{~F}$ & 90 & 4:50 & $1 \mathrm{~B}$ & 4 & PDD & $\mathrm{HC}+\mathrm{SN}$ & Unknown \\
\hline 31 & M & 84 & 9:00 & $1 \mathrm{~A}$ & 5 & PDD & $\mathrm{HC}+\mathrm{SN}$ & Pneunomia and dehydration \\
\hline 32 & $\mathrm{~F}$ & 70 & 7:05 & $2 B$ & 6 & PDD & $\mathrm{HC}+\mathrm{SN}$ & Haematemesis by oesophagitis \\
\hline 33 & $\mathrm{~F}$ & 87 & $5: 25$ & $2 B$ & 6 & PDD & $\mathrm{HC}+\mathrm{SN}$ & Pneunomia \\
\hline 34 & M & 73 & $5: 35$ & $1 \mathrm{~A}$ & 5 & PDD & $\mathrm{HC}+\mathrm{SN}$ & Direct cause unknown (morphine) \\
\hline 35 & M & 83 & 5:15 & 1B & 6 & PDD & $\mathrm{HC}+\mathrm{SN}$ & Pneunomia \\
\hline 36 & $\mathrm{~F}$ & 84 & $7: 25$ & $2 B$ & 5 & PD & $\mathrm{HC}+\mathrm{SN}$ & Old age, shortness of breath \\
\hline 37 & $\mathrm{~F}$ & 96 & $7: 10$ & $1 \mathrm{~B}$ & 5 & PD & $\mathrm{HC}+\mathrm{SN}$ & Old age \\
\hline 38 & M & 86 & $5: 10$ & $2 B$ & 5 & PD & $\mathrm{HC}+\mathrm{SN}$ & Heartfailure \\
\hline 39 & M & 71 & $5: 50$ & $1 \mathrm{~A}$ & 6 & PD & $\mathrm{HC}+\mathrm{SN}$ & Respiratory failure \\
\hline 40 & M & 86 & $5: 35$ & 10 & 4 & PD & SN & Aspiration pneumonia \\
\hline 41 & M & 80 & 7:05 & 1B & 6 & PDD & SN & Unknown, possibly urine tract infection \\
\hline 42 & $\mathrm{~F}$ & 83 & 6:05 & 10 & 4 & PDD & $\mathrm{HC}$ & Cachexia by dementia, infarction \\
\hline 43 & M & 83 & 6:35 & $1 \mathrm{~B}$ & 6 & PDD & $\mathrm{HC}$ & Pneunomia \\
\hline
\end{tabular}

D, clinical diagnosis; PMD, post mortem delay; NFT, neurofibrillary tangles; $A \beta$ amyloid beta; $\alpha$-syn, alpha synucleine; NDC, non-demented control subject; iLBD, incidental Lewy Body Disease cases; PD, Parkinson's disease; PDD, Parkinson's disease with dementia; HC, hippocampus, SN, substantia nigra. 
of $\alpha$-synuclein (Clone KM51, Novacastra, Bioconnect BV), hyperphosphorylated tau (Clone AT8, Pierce, Rockford, IL) and $\mathrm{A} \beta$ (Clone $6 \mathrm{~F} / 3 \mathrm{D}$, DAKO, DakoCytomation $\mathrm{BV})$, respectively. The $\mathrm{AD}$ Braak stages and scores were further matched between control subjects, iLBD cases and PD patients, ruling out any possible differences in microglial activation due to AD pathology. The clinicopathological data of the patients including the Braak staging for PD and AD of all donors is summarized in Table 1.

\section{Tissue processing}

At autopsy, brain regions were dissected and immersionfixed in $4 \%$ formaldehyde for four weeks and subsequently embedded in paraffin. From the paraffin blocks of the $\mathrm{HC}$ and the ventral mesencephalon, which included the SN pars compacta, 10 micrometer $(\mu \mathrm{m})$ thick sections were cut with a microtome, mounted on positivelycharged glass slides (Menzel-Glaser SuperFrost Plus, Braunschweig, Germany) and dried in a stove overnight at $37^{\circ} \mathrm{C}$ before immunohistochemical staining.

\section{Immunohistochemistry}

Sections were heated in a stove for one hour at $56^{\circ} \mathrm{C}$, before they were deparaffinized in xylene and rehydrated through a graded series of ethanol (100\%, 96\%, 90\% and $70 \%$, respectively) and distilled water. For subsequent antigen retrieval, sections were rinsed in $10 \mathrm{mM}$ Tris Buffer (pH 9.0) containing $1 \mathrm{mM}$ EDTA (Tris-EDTA) and placed in preheated Tris-EDTA buffer in a steamer at $90-99^{\circ} \mathrm{C}$ for 30 minutes. For $\alpha$-synuclein staining, antigen retrieval was performed using pretreatment with 98\% formic acid (Sigma, Steinheim, Germany) for $10 \mathrm{~min}$ at room temperature (RT). After pretreatment, the sections were RT, rinsed in Tris-buffered saline (TBS, pH 7.6) and incubated for $20 \mathrm{~min}$ in TBS containing $0.3 \% \mathrm{H}_{2} \mathrm{O}_{2}$ and $0.1 \%$ sodiumazide to block endogenous peroxidase activity. Non-specific binding was blocked with $5 \%$ non-fat dried milk in TBS containing 0.5\% Triton (TBS-T, pH 7.6; blocking solution) for $30 \mathrm{~min}$ at RT. Subsequently, sections were incubated overnight at $4^{\circ} \mathrm{C}$ with mouse antiCD68, mouse anti- $\alpha$-synuclein, goat anti-TLR2 or rabbit anti-Ionized calcium binding adaptor molecule 1 (Iba1) antibodies diluted in blocking solution (details on the primary antibodies are specified in Table 2). Sections were then washed in TBS and incubated for $2 \mathrm{hr}$ at RT in the

Table 2 Primary antibodies used for single labeling

\begin{tabular}{llll}
\hline Antigen & Species & Final dilution & Source \\
\hline Human CD68 & Mouse & $1: 500$ & DAKO, clone KP1 \\
Human a-synuclein & Mouse & $1: 2000$ & BD-Bioscience, 610786 \\
Human Iba1 & Rabbit & $1: 4000$ & WAKO chemicals \\
Human TLR2 & Goat & $1: 2000$ & R\&D systems \\
\hline
\end{tabular}

appropriate dilutions of biotinylated goat anti mouse IgG's, goat anti rabbit IgG's or donkey anti goat IgG's (1:400; Jackson ImmunoResearch Laboratories Inc., West Grove, Pennsylvania, USA) followed by horse radish peroxidase (HRP)-labeled avidin-biotin complex (ABC complex, 1:400; Vector Laboratories, Burlingame, CA, USA) in TBS-T for $1 \mathrm{hr}$ at RT. Iba1 and CD68 staining were visualized using 3,3-diaminobenzidine (DAB, Sigma, St. Louis, USA) and counterstained with heamatoxylin, while TLR2 and $\alpha$-synuclein immunostaining were visualized using DAB-nickel as a chromogen, and counterstaining was performed with Fast red. After dehydration in graded ethanol solutions, sections were cleared in xylene and coverslipped in Entellan (Merck).

\section{Immunofluorescence}

For double-immunofluorescent labeling of glial cells and TLR2 expression, sections were co-incubated with combinations of antibodies against microglia, i.e. CD68, Iba1, or astrocytes, i.e. GFAP, and against TLR2. Antigen retrieval was performed by pretreating the sections with Tris-EDTA (pH 9.0) and all antibodies (TLR2/Iba1, TLR2/CD68, TLR2/GFAP) were diluted in blocking solution, as indicated above. After an overnight incubation at $4^{\circ} \mathrm{C}$, the sections were washed and subsequently incubated for $2 \mathrm{~h}$ at RT with the appropriate Alexa Fluor 488 or Alexa Fluor labeled 594 IgG's (1:400, Jackson Immunoresearch, Westgrove, PA, USA) (see Table 3 for information on antibodies and conjugates). After washing, sections were coverslipped with Vectashield and later examined using a confocal microscope (Leica TSCSP2-AOBS; Leica Microsystems, Wetzlar, Germany).

\section{Microglial phenotypes: identification criteria for ramified, primed/reactive and amoeboid microglia}

Classification of ramified and amoeboid microglia morphology was performed as described in detail before [23]. Microglial cells were identified by positive CD68 immunoreactivity (IR) [53]. Both microglial subtypes are characterized by cytoplasmic staining, but the ramified microglial celltype can be distinguished by its small cell body and thin, radially projecting processes. Amoeboid microglia are characterized by a densely CD68-stained cell body typically surrounded by no or only very few short/stump

Table 3 Antibodies and conjugates used for double labeling

\begin{tabular}{lllll}
\hline Antigen & Species & $\begin{array}{l}\text { Final } \\
\text { dilution }\end{array}$ & Source & $\begin{array}{l}\text { Secondary ab's } \\
\text { and conjugates }\end{array}$ \\
\hline Human Iba1 & Rabbit & 1:2000 & WAKO chemicals & DoaR-AF488 1:400 \\
Cow GFAP & Rabbit & 1:2000 & DAKO & DoaR-AF488 1:400 \\
Human CD68 & Mouse & 1:300 & DAKO, clone KP1 & DoaM-AF488 1:400 \\
Human TLR2 & Goat & $1: 500$ & R\&D systems & DoaG-AF594 1:400 \\
\hline
\end{tabular}


processes [23]. Furthermore, we defined Iba1 positive ramified microglia as cells having a small circular body with highly ramified processes. Iba1 positive microglia with a primed/reactive phenotype display a bigger and less round cell body with thicker and sometimes less ramifications compared to the ramified phenotype. Iba1 positive amoeboid microglia show an amoeboid cell body with, at most, two unramified processes, or they are completely devoid of them [29].

\section{Semi-quantitative analyses of microglial cells, a-synuclein} deposition and TLR2 expression

For semi-quantificative analysis, the numbers of CD68 positive amoeboid microglial cells and $\alpha$-synuclein immunopositive deposits ( $\mathrm{LBs}$ and $\mathrm{LNs}$ ) present in the HC pyramidal cell layers, i.e. CA4, CA3, CA2 and CA1, were counted per CA area (region of interest, ROI, $0.5 \mathrm{~mm}^{2}$ ) at a $10 \times 3.3$ magnification. For standardization purposes, hippocampal sections were collected around the anterior-to-midlevel of the $\mathrm{HC}$ of every subject, and only when large DG and CA subregions were both present. For the SN, the area of interest was larger (ROI, $1.5 \mathrm{~mm}^{2}$ ) and quantification was performed at the anatomical levels of the oculomotor nerve. Microglial cells were counted at a $10 \times 3.3$ magnification; hence, these data are presented as number of cells per $1.5 \mathrm{~mm}^{2}$. Semiquantitative analysis of TLR2 and Iba1 IR was based on the amount of IR per ROI. To this end, pictures were taken at a $10 \times 3.3$ magnification and on these a standardized threshold procedure was used that distinguished background from specific IR. Subsequently, the amount of specific IR was measured within the defined ROI, and expressed as percentages of TLR2 and Iba1 IR per ROI. Semi-quantitative analyses were performed unbiased using Cell $^{\mathrm{F}}$ Olympus Soft Imaging Solutions GmbH software, version 3.1 (Tokyo, Japan). All data were expressed as mean $+/$ - standard error of the mean (SEM).

\section{Statistical analysis}

All statistical analyses were performed with the SPPS package version 20.0 (Statistical Product and Service Solutions, Chicago, IL, USA). The normal distribution of the data was investigated using the Kolmogorov-Smirnov test. When normally distributed (Iba1), statistical analysis for between group effects was performed with an independent sample T-test. When a normal distribution was absent (CD68, TLR2, $\alpha$-synuclein), statistical analyses were executed with the non-parametric Kruskal-Wallis test to examine main group effects between control subjects, iLBD cases and PD patients. Subsequently, MannWhitney $U$ tests were performed as post-hoc tests to assess between group effects. Statistical analyses for the different subregions of the $\mathrm{HC}$ within the same cases were performed with the non-parametric paired Wilcoxon test.
Significance was set at 0.05 with Bonferroni corrections for multiple testing where applicable.

\section{Results}

a-Synuclein pathology and increased numbers of CD68 positive amoeboid microglia in the SN

We first examined the SN that is classically affected in $\mathrm{PD}$. In the SN of control subjects and iLBD cases, numerous neuromelanin-containing and pigmented dopaminergic neurons were present (Figure 1). No $\alpha$-synuclein pathology was observed in the SN of controls, while in the iLBD cases, few $\alpha$-synuclein positive deposits were detected (Figure 1a, b respectively; Figure 2a). In contrast, PD patients showed extensive loss of neuromelanincontaining, dopaminergic neurons in the $\mathrm{SN}$, and the number of LBs and LNs was significantly increased compared to both control and iLBD cases (Figure 1c; Figure 2a; PD vs Ctr/iLBD p $<0.01$; Ctr $n=13$, Mean $=0 \pm 0$; iLBD $n=9$, Mean $=7 \pm 6.2$; PD $n=14$, Mean $=29.2 \pm 4.9$ ). Furthermore, in control subjects, the large majority of CD68 positive microglia had long and fine processes, indicative of their ramified phenotype, with only a small number of cells displaying an amoeboid morphology (Figure 1d). The number of amoeboid microglia was significantly increased in iLBD cases compared to control subjects (Figure 1e; Figure 2b; iLBD vs Ctr $\mathrm{p}=0.002$; Ctr Mean $=13.2 \pm 4.1$; iLBD Mean $=46.6 \pm 12.0$ ). PD patients showed numerous and widespread CD68 positive amoeboid microglia in the $\mathrm{SN}$, significantly more than in the iLBD and in control cases (Figure 1f; Figure 2b; PD vs iLBD $p=0.035$, PD vs Ctr $\mathrm{p}=0.000$; PD Mean $=80.6 \pm 12.9$ ).

\section{a-Synuclein pathology is apparent in the hippocampal CA2 subregion of PD patients}

Next, $\alpha$-synuclein pathology was examined in the $\mathrm{HC}$. No $\alpha$-synuclein IR was present in the $\mathrm{HC}$ of control and iLBD cases (shown for CA2; Figure 3a, b). In contrast, $\alpha$-synuclein positive deposits were prominent in the $\mathrm{HC}$ of PD and PDD patients and especially concentrated in the pyramidal layer CA2, where their numbers were significantly increased compared to the CA2 of control and iLBD cases (Figure 3c; Figure 4b; PD vs Ctr/iLBD $\mathrm{p}<0.01 ; \operatorname{Ctr} \mathrm{n}=9$, Mean $0 \pm 0$; iLBD $\mathrm{n}=6$, Mean $0.5 \pm$ 0.5 ; PD $\mathrm{n}=14$ Mean $24.5 \pm 7.4$ ) and also compared to the other pyramidal regions CA1, CA3 and CA4 within $\mathrm{PD}$ patients (Figure 3c; Figure 4a; significantly different between all regions $\mathrm{p}<0.05$; and CA2 vs CA1/CA3/CA4 $\mathrm{p}=0.001 ;$ CA2 Mean $=24.5 \pm 7.4 ;$ CA1 Mean $=0.8 \pm 0.5$; CA3 Mean $=4.9 \pm 2.5$; CA4 Mean $=3.1 \pm 1.9$ ).

\section{Increased number of CD68 positive amoeboid microglia} in the $\mathrm{HC}$ of iLBD cases and PD patients

In the pyramidal layer of the $\mathrm{HC}$ of control subjects, iLBD cases and PD patients, CD68 positive, ramified 


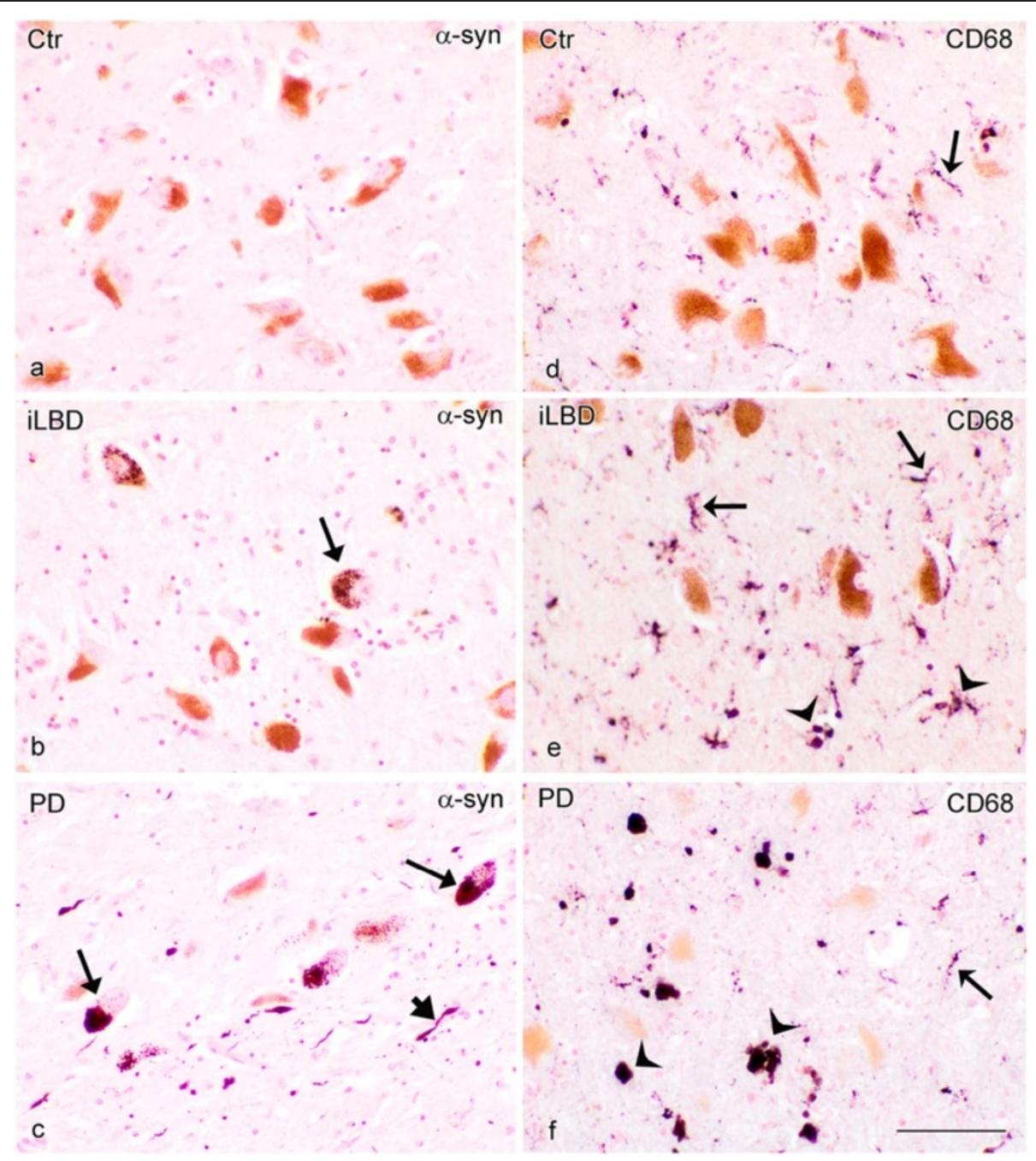

Figure 1 a-Synuclein pathology and CD68 immunopositive microglia in the substantia nigra (SN) of control subjects, iLBD cases and PD patients. Brown staining depicts the melanin-containing, dopaminergic neurons that degenerate during PD. (a) No intraneuronal a-synuclein immunoreactivity (IR)(purple) was observed in the SN of control subjects, (b) some intraneuronal a-synuclein IR was found in the SN of iLBD cases (arrow), and (c) widespread intraneuronal and neuritic a-synuclein IR is observed in the SN of PD patients (LBs: arrows, LNs arrowhead); (d) a control subject showing some CD68 positive ramified microglial cells (purple, arrow), (e) an iLBD case showing prominent CD68 positive ramified (arrow) and amoeboid (concave arrowhead) microglial phenotypes, and (f) a PD patient, showing few CD68 positive ramified (arrow), but many more amoeboid microglial phenotypes (concave arrowhead); bar (a-f) $=100 \mu \mathrm{m}$.

microglia were present (shown for CA2 Figure 3d-f), but CD68 positive amoeboid microglia were clearly more present in PD patients (Figure 3f) compared to control and iLBD cases (Figure 3d, e), especially in the $\mathrm{CA} 2$ and $\mathrm{CA} 3$ region (shown for CA2 Figure $5 \mathrm{~d}$; PD vs Ctr/iLBD $\mathrm{p}=0.007$; Ctr Mean $=13 \pm 2.9$; iLBD Mean $=12.1 \pm 2.5$; PD Mean $=37.2 \pm 7.2$ ). Interestingly, in control and iLBD cases, a significantly higher number of CD68 positive amoeboid microglia was present in the $\mathrm{CA} 2$ and $\mathrm{CA} 3$ as compared to other subregions (Figure 5a, b; Ctr: CA1 vs CA2/CA3/CA4 p < 0.02, CA3 vs CA4 $\mathrm{p}=0.025$; CA1 Mean $=2.7 \pm 0.8$; CA2 Mean $=$ $12.9 \pm 2.9 ;$ CA3 Mean $=14.8 \pm 1.9$; CA4 Mean $=8.5 \pm$
1.9; iLBD: CA1 vs CA2/CA3/CA4 p =0.027, CA4 vs $\mathrm{CA} 2 / \mathrm{CA} 3 \mathrm{p}<0.05$; CA1 Mean $=4.0 \pm 1.0$; $\mathrm{CA} 2$ Mean $=$ $12.2 \pm 2.5 ;$ CA3 Mean $=12.7 \pm 2.0 ;$ CA4 Mean $=7.2 \pm$ 1.4). This subregion-specific difference in CD68 positive amoeboid microglia was larger in PD patients, with the highest numbers present in the CA2 region (Figure $5 \mathrm{c}$; PD: CA1 vs CA2/CA3/CA4 p $<0.01$, CA4 vs CA2/CA3 $\mathrm{p}<0.01$, CA3 vs CA2 $\mathrm{p}=0.278$ n.s.; CA1 Mean $=7.0 \pm$ 1.2; CA2 Mean $=37.2 \pm 7.2 ;$ CA3 Mean $=32.5 \pm 6.5$; CA4 Mean $=17.5 \pm 4.5$ ). Based on the $\alpha$-synuclein and CD68 observations in the $\mathrm{HC}$, our subsequent analyses of Iba1 and TLR2 immunoreactivity focused mainly on the $\mathrm{CA} 2$ region. 

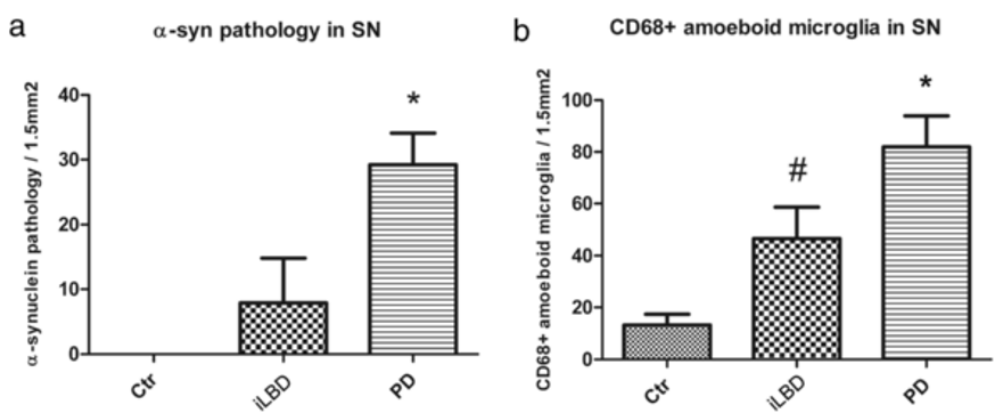

Figure 2 Quantification of a-synuclein pathology and CD68 immunopositive amoeboid microglia density in the substantia nigra (SN) of control subjects, iLBD cases and PD patients. (a) A significant increase was present in a-synuclein deposits in PD patients compared to the control and iLBD cases ( ${ }^{*} p<0.001$ vs Ctr, iLBD), and (b) a significant increase in CD68 positive amoeboid microglia was present in PD patients compared to control subjects and iLBD cases $\left({ }^{*} \mathrm{p}<0.01\right.$ vs $C \mathrm{tr} ;{ }^{*} \mathrm{p}<0.05$ vs iLBD). The number of CD68 positive amoeboid microglia in iLBD cases was also significantly higher compared to control subjects ( $" p<0.01$ vs Ctr). Data represent mean \pm SEM.

\section{Iba1 positive microglia in SN and HC in PD}

In addition to CD68, Iba1 IR was used to determine the overall presence of microglial cells and to classify different phenotypes in the SN and HC. In all subjects, Iba1 positive ramified microglia displayed a small circular cell body with highly ramified processes. This phenotype was the most prominent one in control subjects (Figure 6a, d). In iLBD cases, additional primed/reactive Iba1 positive microglial cells were present, with a bigger and less round cell body and thicker ramifications as compared to the ramified phenotype (Figure 6b, e). These primed/reactive microglia were also present in PD patients with the addition of a few Iba positive amoeboid microglia displaying at most two unramified processes, or none at all (Figure $6 \mathrm{c}, \mathrm{f}$ ).

A significant increase was found in total Iba1 density in the SN of PD patients relative to controls (Figure 6a, c; Figure 7a; PD vs Ctr $\mathrm{p}=0.03$; Ctr Mean $=6.0 \pm 0.6$; PD Mean $=7.7 \pm 0.5$ ), but not in iLBD cases compared to controls. Total Iba1 density did not differ between subregions of the $\mathrm{HC}$ (not shown) nor between control subject, iLBD cases and PD patients (shown for CA2 Figure 6d-f; Figure 7b; Ctr Mean $=7.7 \pm 0.7$; iLBD Mean $=8.3 \pm 1.2$; PD Mean $=7.2 \pm 0.4$ ).

Increased TLR2 expression in the SN and HC of iLBD cases In control subjects, only very few TLR2 positive cells and processes were observed in the SN and HC (Figure 8a, a'; Figure 9a, a'). In iLBD cases, however, TLR2 IR was prominent and widespread throughout the $\mathrm{SN}$ and total $\mathrm{HC}$, and significantly increased relative to control subjects in both regions (Figure 8b, b'; Figure 10; iLBD vs Ctr $\mathrm{p}=0.000 ;$ Ctr Mean $=0.7 \pm 0.2 ;$ iLBD Mean $=5.7 \pm 0.9$; shown for CA2 Figure 9a, b, b'; Figure 11d; iLBD vs Ctr $\mathrm{p}=0.018 ;$ Ctr Mean $=0.7 \pm 0.3 ;$ iLBD Mean $=2.8 \pm 0.7$ ). This upregulation in the $\mathrm{HC}$ was not CA2 specific, but observed in all hippocampal CA regions (Figure 11a-c).
However, within iLBD cases, TLR2 IR was significantly higher in the CA2, CA3 and CA4 as compared to CA1 (Figure 11b; CA1 vs CA2/CA4/CA3 $\mathrm{p}=0.028$; iLBD CA1 Mean $=1.5 \pm 0.4 ;$ CA2 Mean $=2.8 \pm 0.7$; CA3 Mean $=$ $3.8 \pm 0.9$; CA3 Mean $=3.6 \pm 0.8$ ).

In PD, TLR2 expression was significantly decreased compared to iLBD cases in both the SN and HC (SN Figure 8a-c, a'-c'; Figure 10; iLBD vs PD $\mathrm{p}=0.018$; iLBD Mean $=5.7 \pm 0.9 ; \quad$ PD Mean $=3.1 \pm 0.5 ; \quad \mathrm{HC} / \mathrm{CA} 2$ Figure 9a-c, a'-c'; Figure 11d; iLBD vs PD $\mathrm{p}=0.013$; PD Mean $=0.8 \pm 0.2$ ). However, TLR2 IR in the SN of PD patients remained significantly elevated compared to control subjects (Figure 10; Ctr vs PD p $=0.000$; Ctr Mean $=0.7 \pm$ 0.2 ; PD Mean $=3.1 \pm 0.5$ ). In contrast, in all pyramidal layers of the $\mathrm{HC}$ of PD patients, TLR2 IR was low and comparable to the level of control subjects, and TLR2 IR only in the CA2 was slightly but significantly higher relative to the other $\mathrm{CA}$ regions (Figure 11c; CA2 vs CA1/ CA4/CA3 $\mathrm{p}<0.02$; CA2 Mean $=0.8 \pm 0.2$; CA1 Mean $=$ $0.5 \pm 0.2$; CA4 Mean $=0.5 \pm 0.2$; CA3 Mean $=0.7 \pm 0.2$ ).

\section{Colocalization of TLR2 with microglial cells in HC and SN}

To determine which cells express TLR2, double labeling revealed that TLR2 was not expressed by GFAP positive astrocytes in the SN or HC (Figure 12). However, clear colocalization was found between Iba1 positive primed/ reactive microglia and TLR2 IR, in almost all TLR2 positive cells in the HC and SN (Figure 13). Finally, to determine whether amoeboid microglia also express TLR2, double labeling for TLR2 and CD68 revealed that indeed TLR2 IR was present in CD68 positive amoeboid microglia in the SN of PD patients (Figure 14).

\section{Discussion}

In the present study, we investigated different microglial phenotypes, and TLR2 expression in relation to the $\alpha$-synuclein pathology in the SN and HC of iLBD cases, 


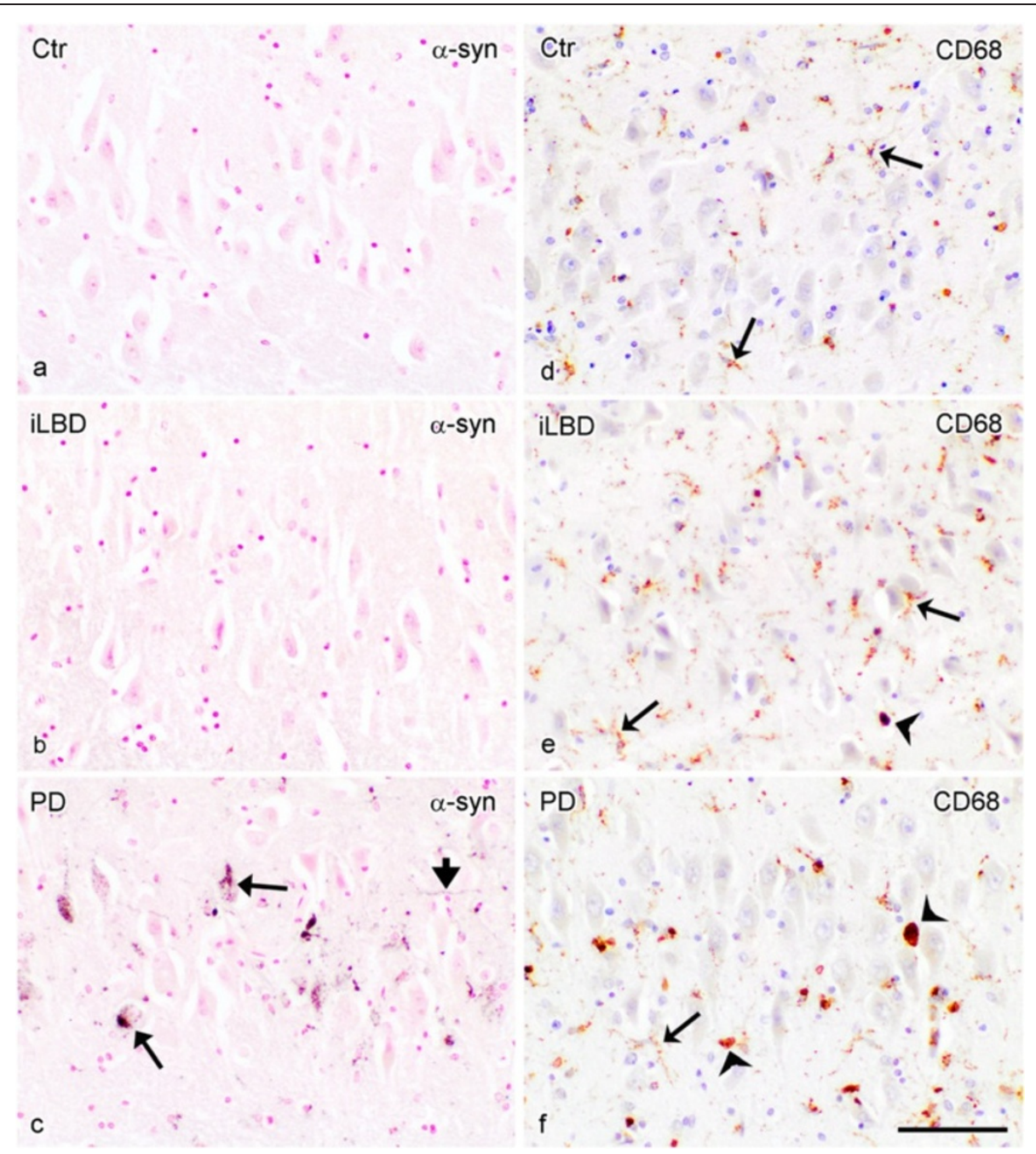

Figure 3 a-Synuclein pathology and CD68 immunopositive microglia in the hippocampal CA2 region of control subjects, iLBD cases and PD patients. $(\mathbf{a}, \mathbf{b})$ a-synuclein IR in the CA2 of control and iLBD cases is absent compared to (c) PD patients (purple, LBs: arrow, LNs: arrowhead); (d) a control subject showing CD68 positive ramified microglial cells (arrow), (e) an iLBD case showing CD68 positive ramified (arrow) and few microglial cells with an amoeboid (concave arrowhead) phenotype and (f) a PD patient, showing CD68 positive ramified (arrow) and several amoeboid (concave arrowhead) microglial cells; bar $(\mathbf{a}-\mathbf{f})=100 \mu \mathrm{m}$.

considered a prodromal state of PD, and of established PD patients. Clear differences were present in microglial phenotypes between the SN and HC. The amoeboid type was found to parallel the prominent $\alpha$-synuclein pathology in the SN and hippocampal CA2 subregion in PD. TLR2 expression was strongly increased in primed/reactive microglia of the iLBD cases. Unexpectedly, in PD patients, TLR2 IR remained upregulated in the $\mathrm{SN}$, but was reduced to control levels in the HC. Thus, TLR2 expression in microglia occurs in a disease stagedependent manner that differs between the $\mathrm{HC}$ and $\mathrm{SN}$. The high levels in iLBD cases and not in PD patients suggest an early activational response to the development of PD pathology. As such, our results confirm, for the first time in human brain, recent in vitro and experimental studies indicating that TLR2 is an important player in the neuroinflammatory responses during PD progression. A general scheme representing the differences in microglial phenotypes and TLR2 expression in the $\mathrm{SN}$ and $\mathrm{HC}$ during disease progression in PD, as represented by the Braak PD stages, is summarized in Figure 15.

In addition to its well established pattern in the $\mathrm{SN}, \alpha-$ synuclein pathology was prominent in the $\mathrm{HC}$, a brain region involved in cognition and affective symptoms that have been implicated in the non-motor symptomatology and frequent dementia and depression in PD patients $[5,7]$. In line with a previous study, $\alpha$-synuclein pathology in the HC was largely confined to the CA2 subregion [54]. We observed that this selective vulnerability 

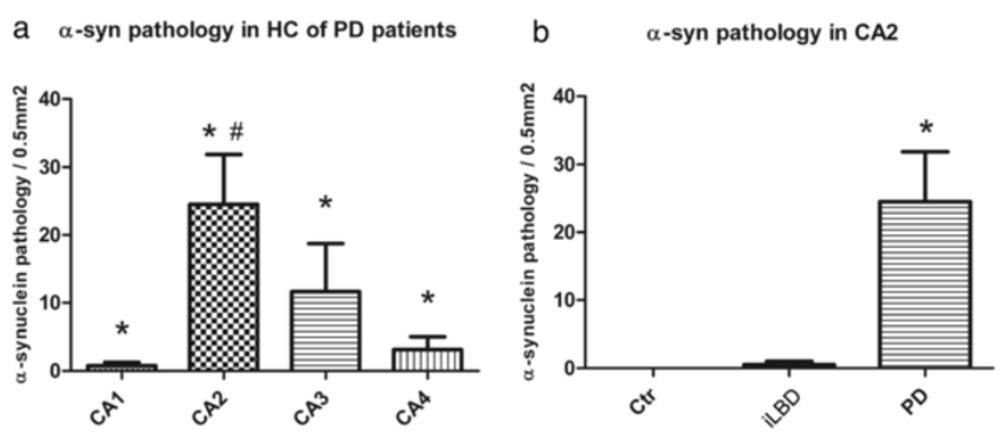

Figure 4 Quantification of a-synuclein pathology in the hippocampus (HC) of control subjects, iLBD cases and PD patients. (a) Significant differences were present in a-synuclein deposits between all pyramidal subregions of the HC in the PD patients $\left({ }^{*} p<0.05\right)$. The CA2 region was affected the most relative to the other hippocampal subregions ( $\left.{ }^{*} p<0.01\right)$. (b) Comparison of a-synuclein pathology in CA2 between the three patient cohorts showed a significant increase in the PD patients compared to control and iLBD cases ( ${ }^{*} p<0.01$ vs iLBD, Ctr). Data represent mean \pm SEM.

was independent of whether PD patients had been diagnosed with dementia. Although only few studies have so far implicated CA2 in PD symptomatology, the selective localization of $\alpha$-synuclein pathology to this subregion and its recent involvement particularly in social forms of memory may hold some promise for a better interpretation of the clinical symptoms $[48,55,56]$.

While general microgliosis has been repeatedly demonstrated in several pathological sites in PD, including the $\mathrm{SN}, \mathrm{HC}$ and $\mathrm{OB}$, little was known about the brain
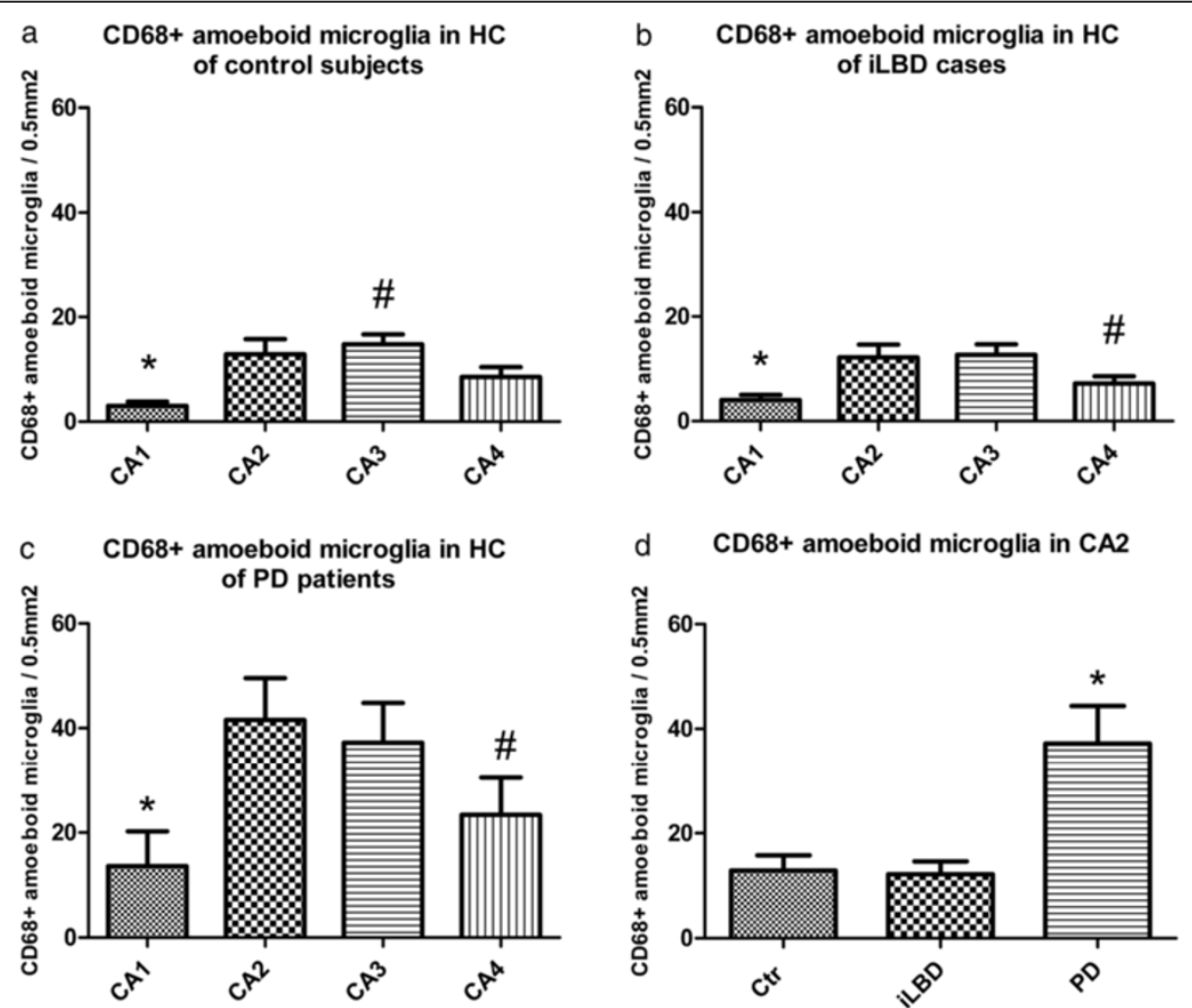

Figure 5 Quantification of CD68 immunopositive amoeboid microglia in the hippocampus (HC) of control subjects, iLBD cases and PD patients. (a-c) Numbers of CD68 positive amoeboid microglia were significantly lower in CA1 compared to all other pyramidal layers in (a) the control subjects, (b) iLBD cases and (c) PD patients ( ${ }^{*} p \leq 0.02$ vs CA2, CA3, CA4). A significant increase was observed in (a) CA3 compared to CA4 of control subjects ( ${ }^{*} p<0.05$ vs CA4), and in (b, c) CA2 and CA3 compared to CA4 in (b) iLBD cases and (c) PD patients $\left({ }^{\#} p<0.05, \# p \leq 0.01\right.$, respectively vs CA2, CA3). (d) Comparison of all groups together resulted in a significant increase in the numbers of CD68 positive amoeboid microglia in the PD patients relative to control and iLBD cases (data shown for CA2, ${ }^{*} p<0.01$ vs iLBD, Ctr). Data represent mean \pm SEM. 


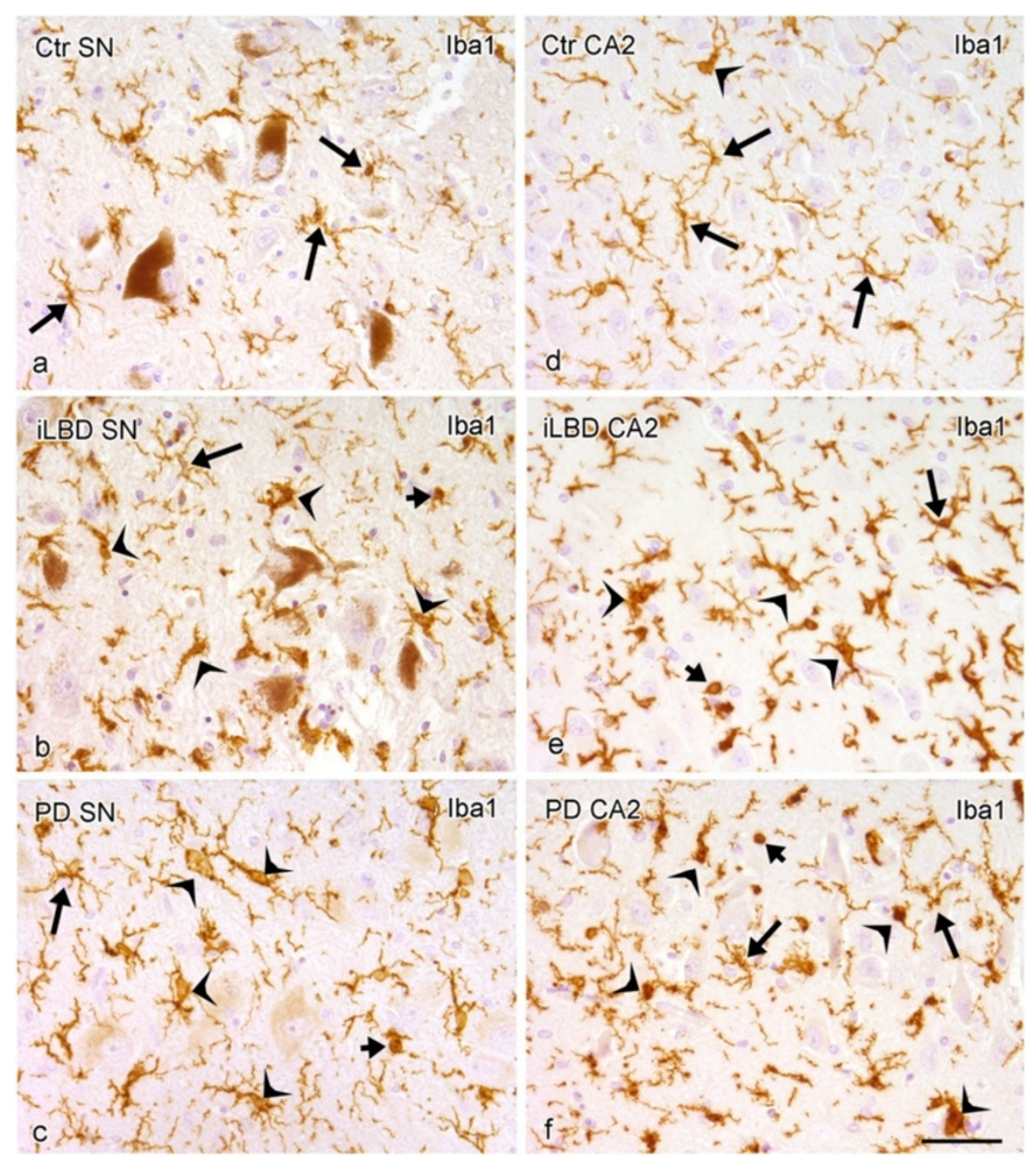

Figure 6 lba1 immunopositive microglia in the substantia nigra (SN) and hippocampal CA2 region of control subjects, iLBD cases and PD patients. (a,b) Widely distributed Iba1 positive ramified microglial cells (arrow) in a control subject, $(\mathbf{c}, \mathbf{d})$ an iLBD case and $(\mathbf{e}, \mathbf{f})$ a PD patient. Iba1 positive microglia are present with ramified (arrow), primed/reactive (concave arrowhead) and amoeboid (short arrow) phenotypes; $\operatorname{bar}(\mathbf{a}-\mathbf{f})=50 \mu \mathrm{m}$.

region-specific phenotypes of microglia and differences between presymptomatic and established PD [23,57,58]. In both the $\mathrm{SN}$ and $\mathrm{HC}$ of $\mathrm{PD}$ patients, the number of activated, CD68 positive, amoeboid microglial cells was increased. As in the $\mathrm{HC}$, this increase was present especially in the CA2 and CA3, notably in close proximity to $\alpha$-synuclein pathology; this suggests that an inflammatory response occurs to these neuropathological alterations, similar to the SN. Strikingly, increases in amoeboid microglia in the SN were observed in iLBD cases too, in which little, if any, DAergic cell loss was present postmortem, nor had any clinical motor symptoms been apparent during these patients' lives [51]. This is of importance since it contributes to the debate whether microglial activation is merely a reaction to neuronal cell death or whether these cell respond to other (early) pathological events [13] and therefore actively participate in the pathological processes before cell death occurs, as suggested by our observations in the $\mathrm{SN}$ and $\mathrm{HC}$. In the $\mathrm{HC}$ however, amoeboid microglia were not increased in iLBD cases, consistent with the fact that the $\mathrm{HC}$ at this stage (Braak PD 1-3) is still devoid of any $\alpha$-synuclein deposits. This is different from the $\mathrm{SN}$ that already shows prominent $\alpha$-synuclein deposits by then. It indicates that $\alpha$-synuclein deposits may trigger a morphological transition into amoeboid microglia before actual cell loss becomes apparent and is in line with observations in the anterior olfactory nucleus [23], and with experimental studies showing that $\alpha$-synuclein can trigger microglial activation $[20,41,47]$. 
a

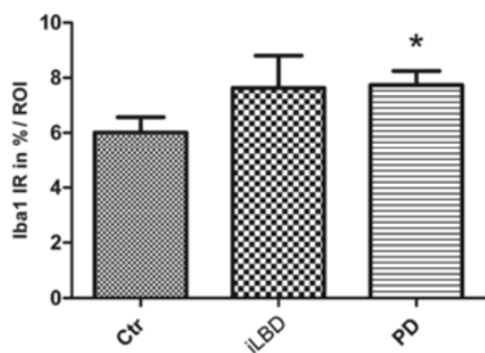

b

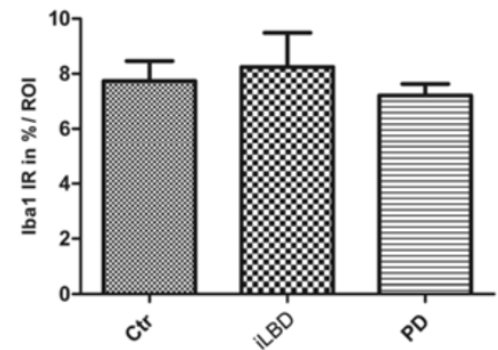

Figure 7 Quantification of Iba1 immunoreactivity (IR) in the substantia nigra (SN) and hippocampal CA2 region of control subjects, iLBD cases and PD patients. (a) A significant increase was present in Ibal IR in the SN of PD patients relative to control subjects ( ${ }^{*} p<0.05$ vs $(t r)$; (b) no significant difference was present in the CA2. Data represent mean \pm SEM.

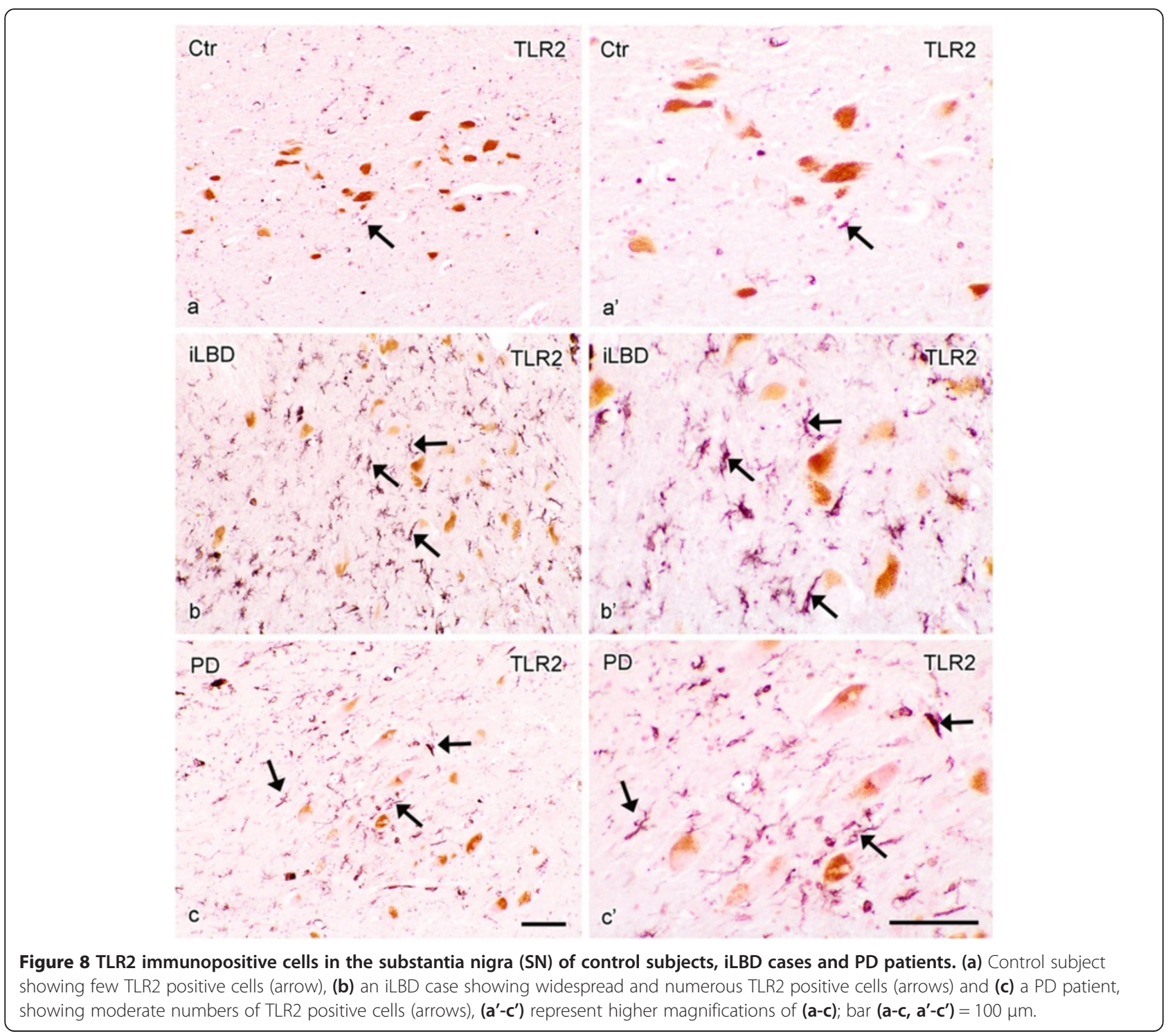




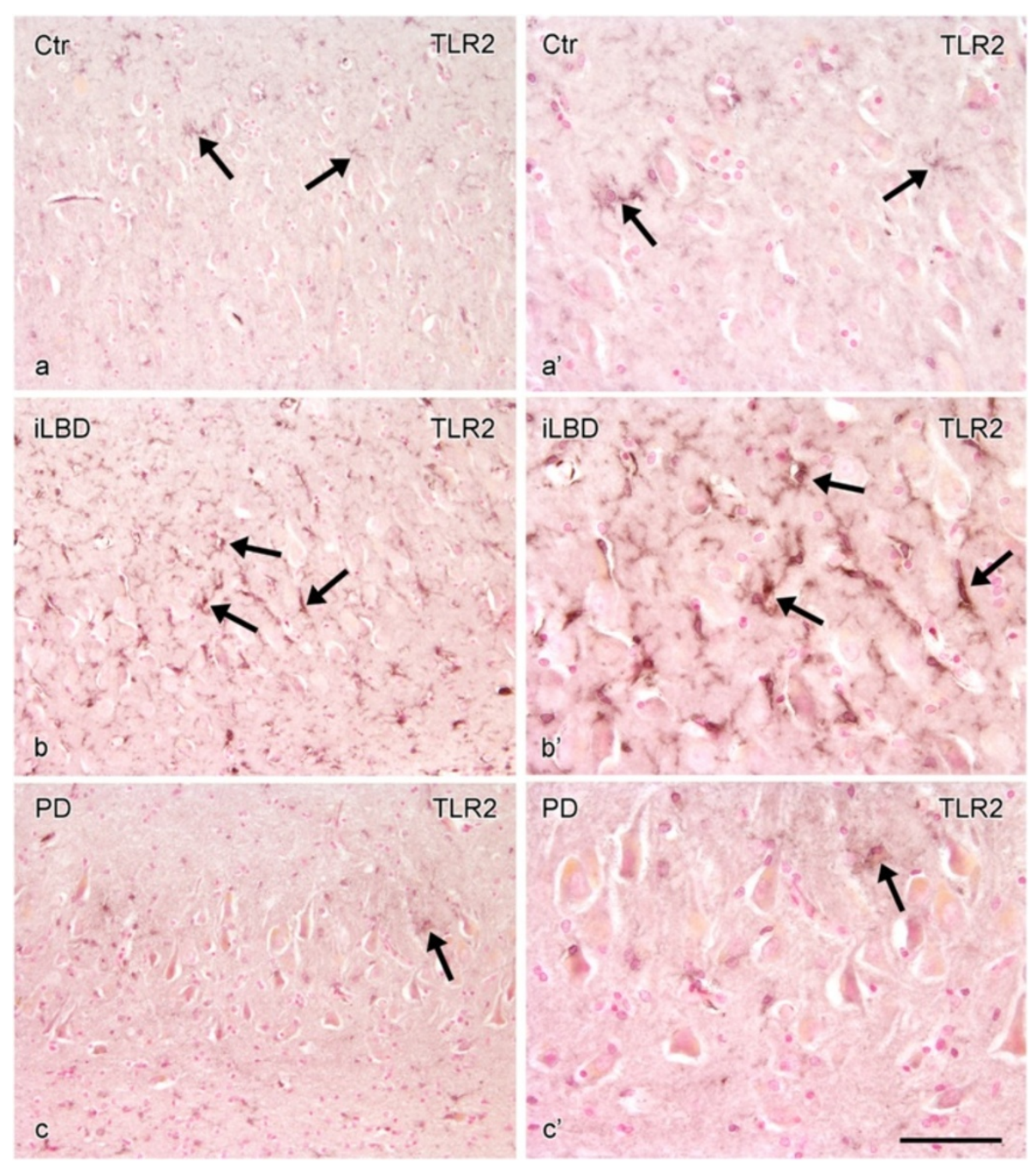

Figure 9 TLR2 immunopositive cells in the hippocampal CA2 region of control subjects, iLBD cases and PD patients. (a) A control subject showing few TLR2 positive cells (arrow), (b) an iLBD case showing widespread and numerous TLR2 positive cells (arrow) and (c) a PD patient, showing again very few TLR2 positive cells (arrow), similar to controls, (a'-c') represent higher magnifications of (a-c); bar $(\mathbf{a}-\mathbf{c})=100 \mu \mathrm{m} ;\left(\mathbf{a}^{\prime}-\mathbf{c}^{\prime}\right)=50 \mu \mathrm{m}$.

Interestingly, in the $\mathrm{HC}$, the total density of Iba1 positive microglia was not different between the subject groups, whereas in the SN an increase was observed in PD patients compared to control subjects. The phenotype of the Iba1 positive ramified microglia had changed into primed/reactive Iba1 positive microglia in the $\mathrm{HC}$ and SN of both the iLBD cases and PD patients, notably irrespective of the presence of $\alpha$-synuclein pathology. Together, this suggests that Iba1 positive ramified microglia may respond to a stimulus other than accumulated or aggregated $\alpha$-synuclein, that likely is independent of the local SN or HC environment and is already present during the prodromal stage. Possibly, the primed microglial morphology represents microglia, which are in a more sensitive state to subsequent stimuli, commonly referred to as 'second hit' [30,31,59]. Although the exact nature of such a stimulus awaits future studies, oligomeric or soluble forms of $\alpha$-synuclein are attractive candidates in this respect.

To further characterize the spatio-temporal changes in microglial phenotypes during PD progression, and to identify possible underlying mechanisms, we focused on TLR2, an important member of the TLR family that was recently implicated in microglial activation in PD $[41,46]$. To rule out any possible differences in microglial activation and TLR2 expression due to AD pathology, NFT and $A \beta$ scores were matched between control subjects, iLBD cases and PD patients. We found TLR2 to be strongly increased in the SN and HC of iLBD cases, but not in PD patients. Recent in vitro and in vivo animal 


\section{TLR2 IR in SN}

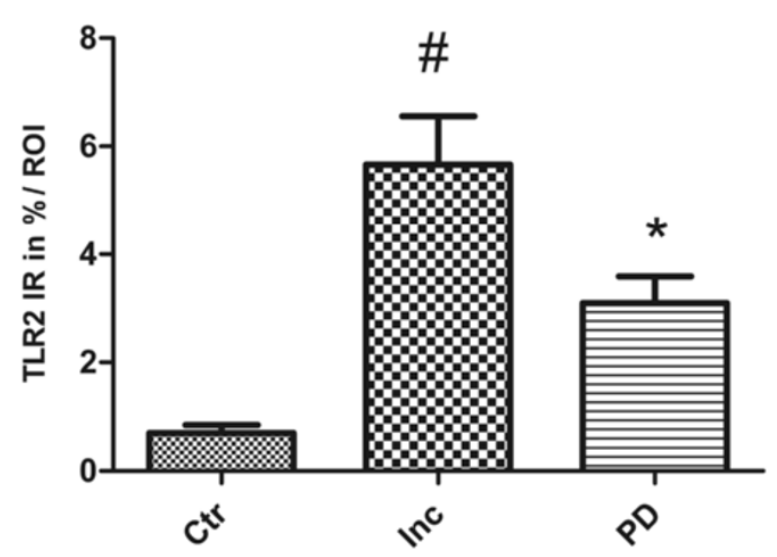

Figure 10 Quantification of TLR2 immunoreactivity (IR) in the substantia nigra (SN) of control subjects, iLBD cases and PD patients. A significant increase was found in TLR2 IR in the SN of iLBD cases compared to control subjects and PD patients (\#p $<0.05$ vs $(t r, P D)$. In PD patients, TLR2 IR was significantly increased in the SN compared to the SN of control subjects $\left({ }^{*} p=0.0001\right.$ vs $\left.C t r\right)$. Data represent mean \pm SEM. studies have shown that $\alpha$-synuclein oligomers can activate microglia via TLR2 and thereby stimulate $\mathrm{NF}_{\mathrm{k}} \mathrm{B}$-mediated pro-inflammatory cytokine production [41,47]. Also, microglial cells treated with $\alpha$-synuclein significantly enhance their TLR2 expression [35]. Since we found the Ibal positive primed/reactive phenotype to express TLR2 particularly [29], this suggests that priming of microglial cells, possibly by $\alpha$-synuclein oligomers, could involve TLR2. The upregulation of TLR2 we found in iLBD cases will then most likely reflect an early activational response of microglia to e.g. $\alpha$-synuclein oligomers, prior to the development of extensive PD pathology, i.e. when neuronal cell death is still barely present. Furthermore, there only seemed to be a topical relation between $\alpha$-synuclein accumulation and TLR2 expression in the SN. In the HC, TLR2 expression was widespread and not confined to the CA2 region, suggesting that the factors causing TLR2 upregulation are present in a more widespread distribution in the $\mathrm{HC}$, consistent with a role for e.g. oligomeric forms of $\alpha$-synuclein. This hypothesis is supported by previous in vitro studies where pre-conditioning microglia with abnormal $\alpha$-synuclein strongly affected their subsequent TLR2 mediated response [44]. A subsequent challenge ('second hit'), like actual $\alpha$-synuclein deposits and/or
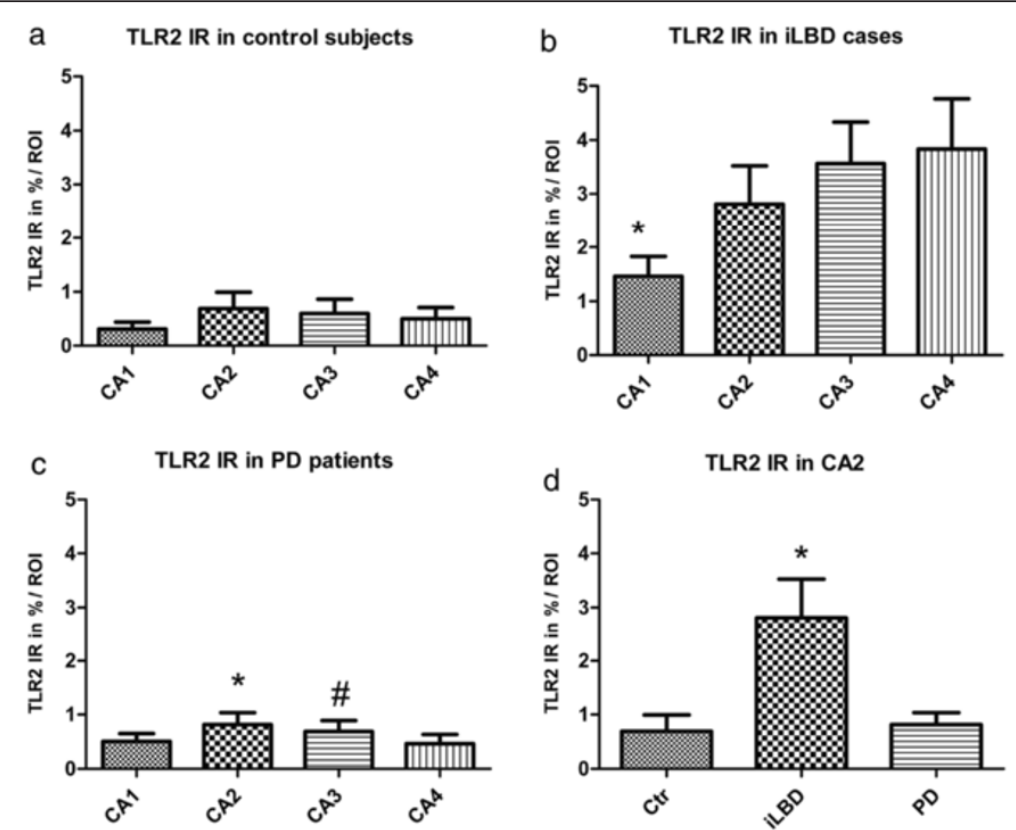

Figure 11 Quantification of TLR2 immunoreactivity (IR) in the hippocampus (HC) of control subjects, iLBD cases and PD patients. (a) No significant differences in TLR2 IR were present between the different hippocampal regions within control subjects, (b) a significant increase in TLR2 IR was found in CA2, CA3 and CA4 compared to CA1 within iLBD cases ( ${ }^{*} p<0.05$ vs CA2, CA3, CA4). (c) In PD patients TLR2 IR was overall found at lower levels that were significantly increased in CA2 relative to the CA1, CA3 and CA4 $\left({ }^{*} p<0.02\right.$ vs CA1, CA3, CA4) and also in CA3 compared to CA4 ( $\mathrm{p} p<0.02$ vs CA4). (d) When TLR2 IR in all four hippocampal subregions was taken together, a significant increase was found in the iLBD cases compared to control subjects and PD patients (data shown for CA2, ${ }^{*} \mathrm{p}<0.02$ ). Data represent mean \pm SEM. 


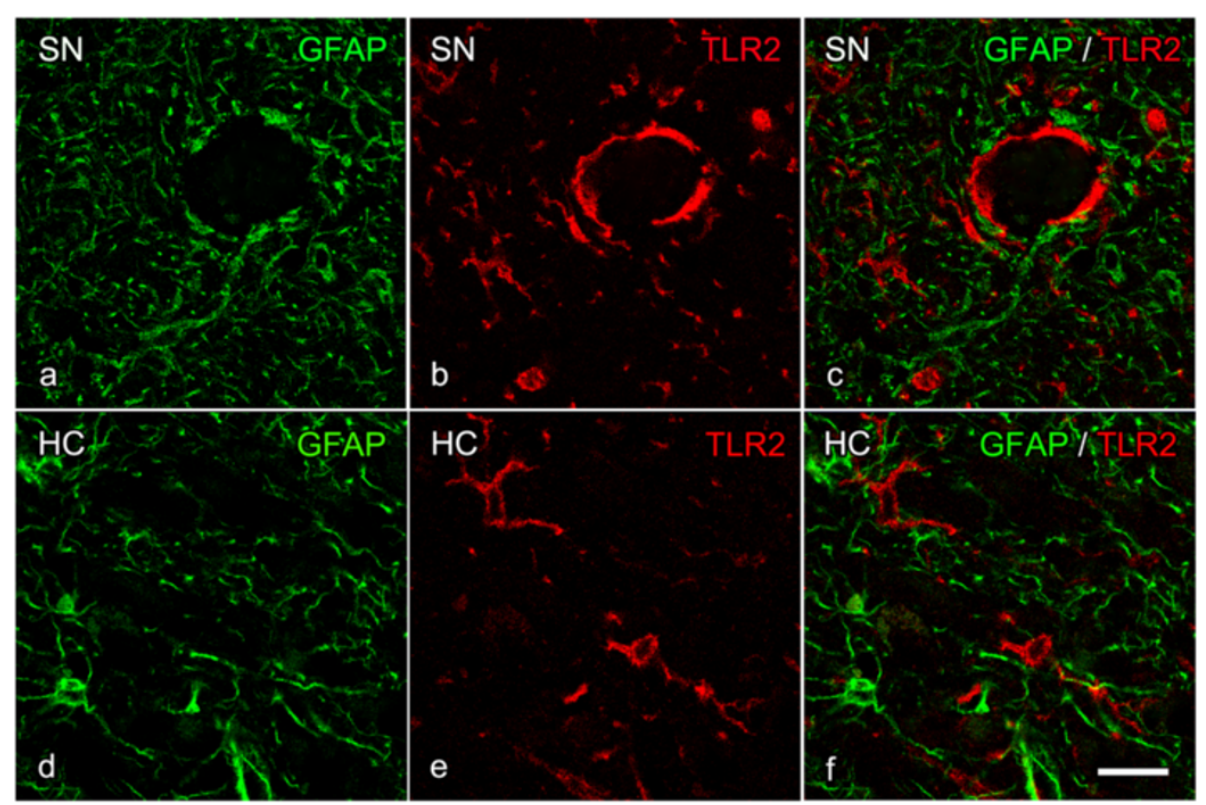

Figure 12 Absence of colocalization of TLR2- and GFAP immunopositive astrocytes in the substantia nigra (SN) and hippocampal CA2 region. (a-f) Representative images of confocal laser scanning microscopy failed to reveal any colocalization (c, $\mathbf{f}$ ) between GFAP (a, $\mathbf{d}$; green) and TLR2 (b, e; red) in the (a-c) SN and (d-f) CA2 of iLBD cases; bar (a-f) $=20 \mu \mathrm{m}$.

DAergic cell loss in the SN of PD patients, could then change the primed phenotype into an amoeboid one, as we observed. This is also in line with the increase in amoeboid microglia in CA2 and CA3, where $\alpha$-synuclein deposits are abundant.
In the HC of PD patients, TLR2 IR was reduced to the level of control subjects, whereas in the $\mathrm{SN}$, its expression remained significantly increased. This difference in expression may reflect the absence of a continuous stimulus, like neuronal loss, that is not apparent in the
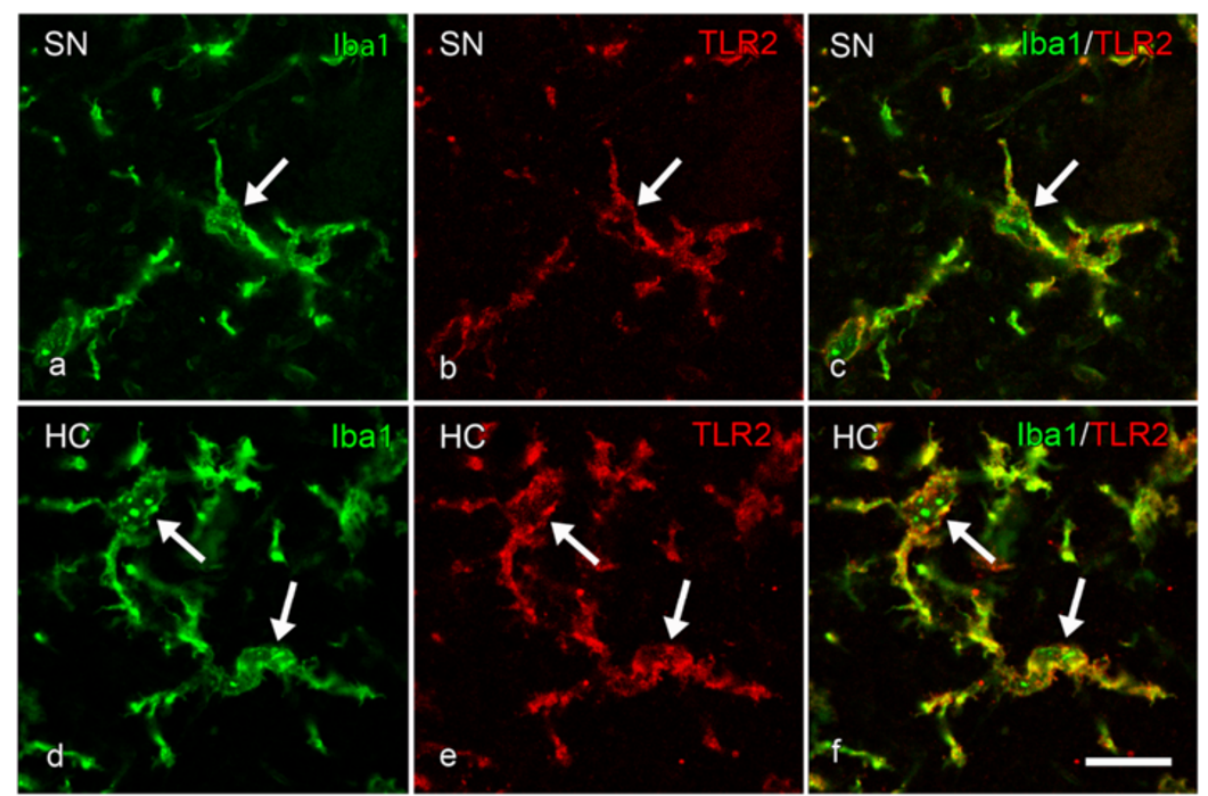

Figure 13 Colocalization of TLR2- and lba1 immunopositive primed/reactive microglia in the substantia nigra (SN) and hippocampal CA2 region of iLBD cases. (a-f) Representative images of confocal laser scanning microscopy revealed colocalization (arrow; $\mathbf{c}, \mathbf{f}$ ) of Iba1 positive primed/reactive microglia (a, d; green) and TLR2 (b, e; red) in the (a-c) SN and (d-f) CA2 of iLBD cases; bar (a-f) $=10 \mu \mathrm{m}$. 

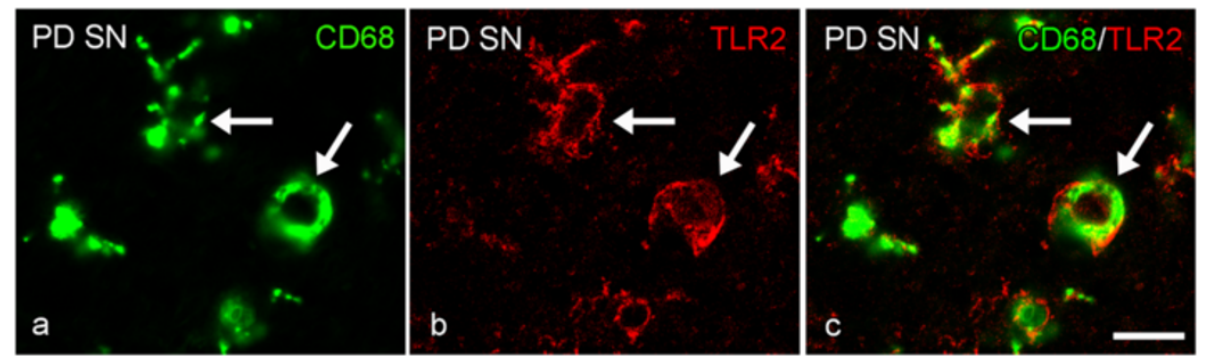

Figure 14 Colocalization of TLR2- and CD68 immunopositive amoeboid microglia in the substantia nigra (SN) of PD patients. (a-c) Representative images of confocal laser scanning microscopical images revealed colocalization (arrow; $\mathbf{c}$ ) of CD68 positive amoeboid microglia (a; green) and TLR2 (b; red) in the SN of PD patients; bar $(\mathbf{a}-\mathbf{f})=10 \mu \mathrm{m}$.

$\mathrm{HC}$, but does occur in the SN. Indeed, whereas some hippocampal atrophy has been observed by MRI [60,61], neuronal loss is not present in the $\mathrm{HC}$ of PD patients [62]. Interestingly, in the CA2, TLR2 expression was still significantly elevated. This also supports the idea that a second stimulus, e.g. $\alpha$-synuclein deposits, after an early pathological stimulus in iLBD cases, may further trigger TLR2 and microglial activation in PD.

Although the primary role of microglia may be to clear toxic proteins and thereby protect neurons, TLR-2 mediated activated microglia have taken on different or additional roles in chronically diseased brains. So far, most studies interpret microglial activation by TLR2 as a classical microglial activation profile that gives rise to the secretion of mainly pro-inflammatory cytokines $[41,63,64]$. However, other studies suggest that receptors like TLR2 can be considered as a 'gateway' in their functionality that can influence the balance between e.g. phagocytic and pro-inflammatory microglial activity [63]. A high expression level of such receptors would then promote an alternative route of activation (e.g. an anti-inflammatory

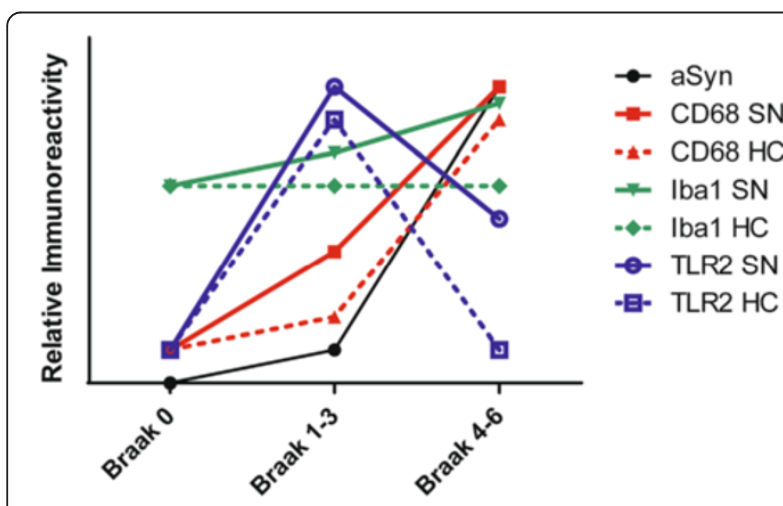

Figure 15 Schematic summary of the different patterns of a-synuclein pathology and microglial phenotypes and activation (Iba1, CD68, TLR2) in the hippocampal CA2 region and SN during disease progression in PD. profile) and phagocytosis, whereas low levels could induce a pro-inflammatory state. It is as yet unknown whether this concept would also apply to TLR2 expression in PD, but it could have important implications for therapy [65]. The final consequences of the TLR2 activation we report here may however, also depend on additional factors, like the assembly state of the protein ligand, the duration of stimulation and the repertoire of co-receptor and adaptor proteins that interact with TLR2 [41].

In summary, we report region-specific differences in the expression of different microglial phenotypes in the $\mathrm{SN}$, a classically affected brain region involved in motor symptoms in $\mathrm{PD}$, and in the $\mathrm{HC}$, a brain region relevant for some of the non-motor symptoms, like dementia, in PD. Besides differential changes in microglial activation and phenotypes in the SN and hippocampal CA2 of PD patients, TLR2 is strongly expressed in primed/reactive microglia in iLBD cases, considered a prodromal state of $\mathrm{PD}$, in both the $\mathrm{HC}$ and SN. Understanding how microglia responses and activation differs between regions and how they change during disease progression will improve our understanding of the role of microglia in neurodegeneration and neuroprotection in general, and of their role in PD pathology in particular. Since neuroinflammatory responses are in principle modifiable, such approaches may help to develop new drugable targets or adjunctive therapies for PD-related symptoms.

\footnotetext{
Abbreviations

PD: Parkinson's disease; AD: Alzheimer's disease; MS: Multiple sclerosis; SN: Substantia nigra; HC: Hippocampus; CA: Cornus ammonis; OB: Olfactory bulb; AON: Anterior olfactory nucleus; IL: Interleukin; iLBD: Incidental Lewy body disease; LBs: Lewy bodies; LN: Lewy neurites; a-synuclein: Alpha-synuclein; AB: Amyloid-beta; NFT: Neurofibrillary tangles; TLR: Toll like receptor; TNFa: Tumor necrosis factor-alpha; MPTP: 1-methyl-4-phenyl-1,2,3, 6-tetrahydropyridinel; Iba1: Ionized calcium binding adaptor molecule 1; GFAP: Glial fibrillary acidic protein; HPtau: Hyperphosphorylated tau; IR: Immunoreactivity.
} 


\section{Acknowledgements}

We thank the Netherlands Brain Bank (Amsterdam, The Netherlands) and Dr. I. Huitinga for provision and help with the human brain tissue. PJL, KJD, WvdB and AMvD are financially supported by the Stichting Parkinson Fonds (SPF), PJL is supported by Alzheimer Nederland and Internationale Stichting Alzheimer Onderzoek ( ISAO).

\section{Author details}

${ }^{1}$ Swammerdam Institute for Life Sciences, Center for Neuroscience, University of Amsterdam, Science Park 904, 1098 XH, Amsterdam, The Netherlands. ${ }^{2}$ Department Anatomy and Neurosciences, VU University Medical Center, Neuroscience Campus Amsterdam, Van der Boechorststraat 7, 1081 BT, Amsterdam, The Netherlands.

\section{Received: 14 July 2014 Accepted: 19 July 2014} Published: 7 August 2014

\section{References}

1. Alves G, Forsaa EB, Pedersen KF, Dreetz Gjerstad M, Larsen JP (2008) Epidemiology of Parkinson's disease. J Neurol 255:18-32

2. Dauer W, Przedborski S (2003) Parkinson's disease: mechanisms and models. Neuron 39:889-909

3. Gaenslen A, Swid I, Liepelt Scarfone I, Godau J, Berg D (2011) The patients' perception of prodromal symptoms before the initial diagnosis of Parkinson's disease. Mov Disord 26:653-658

4. Dickson DW, Fujishiro H, Orr C, DelleDonne A, Josephs KA, Frigerio R, Burnett M, Parisi JE, Klos KJ, Ahlskog JE (2009) Neuropathology of non-motor features of Parkinson disease. Parkinsonism Relat Disord 15:S1-S5

5. Reichmann H, Schneider C, Löhle M (2009) Non-motor features of Parkinson's disease: depression and dementia. Parkinsonism Relat Disord 15:S87-S92

6. Grinberg LT, Rueb U, Alho ATL, Heinsen H (2010) Brainstem pathology and non-motor symptoms in PD. J Neurol Sci 289:81-88

7. Aarsland D, Kurz MW (2010) The epidemiology of dementia associated with Parkinson's disease. Brain Pathol 20:633-639

8. Halliday A, Barker RA, Rowe DB (2011) Non-dopamine Lesions in Parkinson's Disease. Oxford University Press, Inc., New York

9. Braak H, Tredici KD, Rüb U, de Vos RAl, Jansen Steur ENH, Braak E (2003) Staging of brain pathology related to sporadic Parkinson's disease. Neurobiol Aging 24:197-211

10. Braak H, Del Tredici K, Bratzke H, Hamm-Clement J, Sandmann-Keil D, Rüb U (2002) Staging of the intracerebral inclusion body pathology associated with idiopathic Parkinson's disease (preclinical and clinical stages). J Neurol 249:1-5

11. Zhang W, Wang T, Pei Z, Miller DS, Wu X, Block ML, Wilson B, Zhang W, Zhou Y, Hong JS, Zhang J (2005) Aggregated alpha-synuclein activates microglia: a process leading to disease progression in Parkinson's disease. FASEB J 19:533-542

12. Wu DC, Jackson-Lewis V, Vila M, Tieu K, Teismann P, Vadseth C, Choi DK, Ischiropoulos H, Przedborski S (2002) Blockade of microglial activation is neuroprotective in the 1-methyl-4-phenyl-1, 2, 3, 6-tetrahydropyridine mouse model of Parkinson disease. J Neurosci 22:1763-1771

13. Doorn KJ, Lucassen PJ, Boddeke HW, Prins M, Berendse HW, Drukarch B, Van Dam AM (2012) Emerging roles of microglial activation and non-motor symptoms in Parkinson's disease. Prog Neurobiol 98:222-238

14. Kreutzberg GW (1996) Microglia: a sensor for pathological events in the CNS. Trends Neurosci 19:312-318

15. Walter L, Neumann H (2009) Role of microglia in neuronal degeneration and regeneration. Semin Immunopath 31:513-525

16. Mogi M, Harada M, Kondo T, Riederer $P$, Inagaki H, Minami M, Nagatsu T (1994) Interleukin-1 [beta], interleukin-6, epidermal growth factor and transforming growth factor-[alpha] are elevated in the brain from Parkinsonian patients. Neurosci Lett 180:147-150

17. Ouchi Y, Yagi S, Yokokura M, Sakamoto M (2009) Neuroinflammation in the living brain of Parkinson's disease. Parkinsonism Relat Disord 15:200-204

18. Gerhard A, Pavese N, Hotton G, Turkheimer F, Es M, Hammers A, Eggert K, Oertel W, Banati RB, Brooks DJ (2006) In vivo imaging of microglial activation with [11C](R)-PK11195 PET in idiopathic Parkinson's disease. Neurobiol Dis 21:404-412

19. McGeer PL, McGeer EG (2008) Glial reactions in Parkinson's disease. Mov Disord 23:474-483
20. Su X, Maguire-Zeiss KA, Giuliano R, Prifti L, Venkatesh K, Federoff HJ (2008) Synuclein activates microglia in a model of Parkinson's disease. Neurobiol Aging 29:1690-1701

21. Tissingh G, Berendse HW, Bergmans P, DeWaard R, Drukarch B, Stoof JC, Wolters EC (2001) Loss of olfaction in de novo and treated Parkinson's disease: possible implications for early diagnosis. Mov Disord 16:41-46

22. Doty RL, Hawkes CH, Berendse HW (2011) Olfactory Dysfunction in Parkinson's Disease and Related Disorders. In: Halliday GM, Barker RA, Rowe DB (eds) Non-dopamine Lesions in Parkinson's Disease., pp 65-91

23. Doorn KJ, Goudriaan A, Blits-Huizinga C, Bol JGJM, Rozemuller AJ, Hoogland PVJM, Lucassen PJ, Drukarch B, Van de Berg WDJ, Van Dam AM (2014) Increased amoeboid microglial density in the olfactory bulb of Parkinson's and Alzheimer's patients. Brain Pathol 24:152-165

24. Vroon A, Drukarch B, Bol JGJM, Cras P, Brevé JJP, Allan SM, Relton JK, Hoogland PVJM, Van Dam AM (2007) Neuroinflammation in Parkinson's patients and MPTP-treated mice is not restricted to the nigrostriatal system: microgliosis and differential expression of interleukin-1 receptors in the olfactory bulb. Exp Gerontol 42:762-771

25. Schwartz M, Butovsky O, Bruck W, Hanisch UK (2006) Microglial phenotype: is the commitment reversible? Trends Neurosci 29:68-74

26. Hanisch UK, Kettenmann H (2007) Microglia: active sensor and versatile effector cells in the normal and pathologic brain. Nat Neurosci 10:1387-1394

27. De Haas AH, Boddeke HW, Biber K (2008) Region specific expression of immunoregulatory proteins on microglia in the healthy CNS. Glia 56:888-894

28. Stence N, Waite M, Dailey ME (2001) Dynamics of microglial activation: a confocal time lapse analysis in hippocampal slices. Glia 33:256-266

29. Torres-Platas SG, Comeau S, Rachalski A, Dal Bo G, Cruceanu C, Turecki G, Giros B, Mechawar N (2014) Morphometric characterization of microglial phenotypes in human cerebral cortex. J Neuroinflam 11:12

30. Sheng JG, Mrak RE, Griffin WS (1997) Neuritic plaque evolution in Alzheimer's disease is accompanied by transition of activated microglia from primed to enlarged to phagocytic forms. Acta Neuropathol 94:1-5

31. Perry VH, Holmes C (2014) Microglial priming in neurodegenerative disease. Nat Rev Neurol 10(4):217-224

32. Norden DM, Godbout JP (2013) Review: microglia of the aged brain: primed to be activated and resistant to regulation. Neuropathol Appl Neurobiol 39:19-34

33. Palm NW, Medzhitov R (2009) Pattern recognition receptors and control of adaptive immunity. Immunol Rev 227:221-233

34. Sloane JA, Blitz D, Margolin Z, Vartanian T (2010) A clear and present danger: endogenous ligands of Toll-like receptors. Neruomol Med 12:149-163

35. Béraud D, Twomey M, Bloom B, Mittereder A, Ton V, Neitzke K, Chasovskikh S, Mhyre TR, Maguire-Zeiss KA (2011) a-Synuclein alters toll-like receptor expression. Front Neurosci 5:80

36. Trudler D, Farfara D, Frenkel D (2010) Toll-like receptors expression and signaling in glia cells in neuro-amyloidogenic diseases: towards future therapeutic application. Mediators Inflamm doi:10.1155/2010/497987

37. Lehnardt S (2010) Innate immunity and neuroinflammation in the CNS: the role of microglia in toll-like receptor-mediated neuronal injury. Glia 58:253-263

38. Walter S, Letiembre M, Liu Y, Heine H, Penke B, Hao W, Bode B, Manietta N, Walter J, Schulz-Schuffer W, Fassbender K (2007) Role of the toll-like receptor 4 in neuroinflammation in Alzheimer's disease. Cell Physiol Biochem 20:947-956

39. Jana M, Palencia CA, Pahan K (2008) Fibrillar amyloid-b peptides activate microglia via TLR2: implications for Alzheimer's disease. J Immunol 181:7254-7262

40. Racke MK, Drew PD (2009) Toll-like receptors in multiple sclerosis. Curr Top Microbiol Immunol 336:155-168

41. Kim C, Ho DH, Suk JE, You S, Michael S, Kang J, Lee SJ, Masliah E, Hwang D, Lee HJ (2013) Neuron-released oligomeric a-synuclein is an endogenous agonist of TLR2 for paracrine activation of microglia. Nat Commun 4:1562

42. Letiembre M, Liu Y, Walter S, Hao W, Pfander T, Wrede A, Schulz-Schaeffer W, Fassbender K (2009) Screening of innate immune receptors in neurodegenerative diseases: a similar pattern. Neurobiol Aging 30:759-768

43. Béraud D, Maguire-Zeiss KA (2012) Misfolded a-synuclein and toll-like receptors: therapeutic targets for Parkinson's disease. Parkinsonism Relat Disord 18:S17-S20

44. Roodveldt C, Labrador-Garrido A, Gonzalez-Rey E, Lachaud CC, Guilliams T, Fernandez-Montesinos R, Itez-Rondan A, Robledo G, Hmadcha A, Delgado M, Dobson CM, Pozo D (2013) Preconditioning of microglia by a-synuclein 
strongly affects the response induced by toll-like receptor (TLR) stimulation. PLOS ONE 8:e79160

45. Noelker C, Morel L, Lescot T, Osterloh A, varez-Fischer D, Breloer M, Henze C, Depboylu C, Skrzydelski D, Michel PP, Dodel RC, Lu L, Hirsch EC, Hunot S, Hartman A (2013) Toll like receptor 4 mediates cell death in a mouse MPTP model of Parkinson disease. Sci Rep 3:1393

46. Watson MB, Richter F, Lee SK, Gabby L, Wu J, Masliah E, Effros RB, Chesselet MF (2012) Regionally-specific microglial activation in young mice over-expressing human wildtype alpha-synuclein. Exp Neurol 237:318-334

47. Codolo G, Plotegher N, Pozzobon T, Brucale M, Tessari I, Bubacco L, de Bernard M (2013) Triggering of inflammasome by aggregated A-synuclein, an inflammatory response in synucleinopathies. PLoS ONE 8:e55375

48. Lim Y, Kehm VM, Lee EB, Soper JH, Li C, Trojanowski JQ, Lee VMY (2011) Alpha-syn suppression reverses synaptic and memory defects in a mouse model of dementia with Lewy bodies. J Neurosci 31:10076-10087

49. Carlesimo GA, Piras F, Assogna F, Pontieri FE, Caltagirone C, Spalletta G (2012) Hippocampal abnormalities and memory deficits in Parkinson disease A multimodal imaging study. Neurology 78:1939-1945

50. Braak H, Ghebremedhin E, Rüb U, Bratzke H, Del Tredici K (2004) Stages in the development of Parkinson's disease-related pathology. Cell Tissue Res 318:121-134

51. van de Berg WDJ, Hepp DH, Dijkstra AA, Rozemuller JA, Berendse HW, Foncke E (2012) Patterns of alpha-synuclein pathology in incidental cases and clinical subtypes of Parkinson's disease. Parkinsonism Relat Disord 18:S28-S30

52. Alafuzoff I, Ince PG, Arzberger T, Al-Sarraj S, Bell J, Bodi I, Bogdanovic N, Bugiani O, Ferrer I, Gelpi E, Gentleman S, Giaccone G, Ironside JW, Kavantzas N, King A, Korkolopoulou P, Kovacs GG, Meyronet D, Monoranu C, Parchi P, Parkkinen L, Patsouris E, Roggendorf W, Rozemuller A, Stadelmann-Nessler C, Streichenberger N, Thal DR, Kretzschmar H (2009) Staging/typing of Lewy body related alpha-synuclein pathology: a study of the BrainNet Europe Consortium. Acta Neuropathol 117:635-652

53. Holness CL, Simmons DL (1993) Molecular cloning of CD68, a human macrophage marker related to lysosomal glycoproteins. Blood 81:1607-1613

54. Armstrong RA, Kotzbauer PT, Perlmutter JS, Campbell MC, Hurth KM, Schmidt RE, Cairns NJ (2014) A quantitative study of a-synuclein pathology in fifteen cases of dementia associated with Parkinson disease. J Neural Transm 121:171-181

55. Pereira JB, Junqué C, Bartrès-Faz D, Ramírez-Ruiz B, Marti MJ, Tolosa E (2013) Regional vulnerability of hippocampal subfields and memory deficits in Parkinson's disease. Hippocampus 8:720-728

56. Hitti FL, Siegelbaum SA (2014) The hippocampal CA2 region is essential for social memory. Nature 508:88-92

57. McGeer PL, Itagaki S, Boyes BE, McGeer EG (1988) Reactive microglia are positive for HLA-DR in the substantia nigra of Parkinson's and Alzheimer's disease brains. Neurology 38:1285-1286

58. Imamura K, Hishikawa N, Ono K, Suzuki H, Sawada M, Nagatsu T, Yoshida M, Hashizume Y (2005) Cytokine production of activated microglia and decrease in neurotrophic factors of neurons in the hippocampus of Lewy body disease brains. Acta Neuropathol 109:141-150

59. Perry VH (2010) Contribution of systemic inflammation to chronic neurodegeneration. Acta Neuropathol 120:277-286

60. Ibarretxe Bilbao N, Tolosa E, Junque C, Marti MJ (2009) MRI and cognitive impairment in Parkinson's disease. Mov Disord 24:S748-S753

61. Melzer TR, Watts R, MacAskill MR, Pitcher TL, Livingston L, Keenan RJ, Dalrymple-Alford JC, Anderson TJ (2012) Grey matter atrophy in cognitively impaired Parkinson's disease. J Neurol Neurosurg Psy 83:188-194

62. Joelving FC, Billeskov R, Christensen JR, West M, Pakkenberg B (2006) Hippocampal neuron and glial cell numbers in Parkinson's disease: a stereological study. Hippocampus 16:826-833

63. Boche D, Perry VH, Nicoll JA (2013) Review: activation patterns of microglia and their identification in the human brain. Neuropathol Appl Eurobiol $39: 3-18$
64. Mantovani A, Sica A, Sozzani S, Allavena P, Vecchi A, Locati M (2004) The chemokine system in diverse forms of macrophage activation and polarization. Trends Immunol 25:677-686

65. Frank S, Burbach GJ, Bonin M, Walter M, Streit W, Bechmann I, Deller T (2008) TREM2 is upregulated in amyloid plaque-associated microglia in aged APP23 transgenic mice. Glia 56:1438-1447

doi:10.1186/s40478-014-0090-

Cite this article as: Doorn et al:: Microglial phenotypes and toll-like receptor 2 in the substantia nigra and hippocampus of incidental Lewy body disease cases and Parkinson's disease patients. Acta Neuropathologica Communications 2014 2:90.

\section{Submit your next manuscript to BioMed Central and take full advantage of:}

- Convenient online submission

- Thorough peer review

- No space constraints or color figure charges

- Immediate publication on acceptance

- Inclusion in PubMed, CAS, Scopus and Google Scholar

- Research which is freely available for redistribution

Submit your manuscript at www.biomedcentral.com/submit
Ciomed Central 